\title{
SPARSE SOLUTIONS IN OPTIMAL CONTROL OF PDES WITH UNCERTAIN PARAMETERS: THE LINEAR CASE*
}

\author{
CHEN LI ${ }^{\dagger}$ AND GEORG STADLER ${ }^{\dagger}$
}

\begin{abstract}
We study sparse solutions of optimal control problems governed by PDEs with uncertain coefficients. We propose two formulations, one where the solution is a deterministic control optimizing the mean objective, and a formulation aiming at stochastic controls that share the same sparsity structure. In both formulations, regions where the controls do not vanish can be interpreted as optimal locations for placing control devices. In this paper, we focus on linear PDEs with linearly entering uncertain parameters. Under these assumptions, the deterministic formulation reduces to a problem with known structure, and thus we mainly focus on the stochastic control formulation. Here, shared sparsity is achieved by incorporating the $L^{1}$-norm of the mean of the pointwise squared controls in the objective. We reformulate the problem using a norm reweighting function that is defined over physical space only and thus helps to avoid approximation of the random space using samples or quadrature. We show that a fixed point algorithm applied to the norm reweighting formulation leads to a variant of the well-studied iterative reweighted least squares (IRLS) algorithm, and we propose a novel preconditioned Newton-conjugate gradient method to speed up the IRLS algorithm. We combine our algorithms with low-rank operator approximations, for which we provide estimates of the truncation error. We carefully examine the computational complexity of the resulting algorithms. The sparsity structure of the optimal controls and the performance of the solution algorithms are studied numerically using control problems governed by the Laplace and Helmholtz equations. In these experiments the Newton variant clearly outperforms the IRLS method.
\end{abstract}

Key words. Optimal control of PDEs, uncertainty, sparse controls, iterative reweighting, $L^{1}$ minimization, Newton method

AMS subject classifications. 60H35, 35Q93, 35R60, 49M15, 49J52

1. Introduction. Solving optimal control problems governed by partial differential equations (PDEs) that contain uncertain parameters represents a significant challenge. However, thanks to theoretical and algorithmic advances, and to the ever increasing availability of computing resources, significant progress has been made over the last decade $[2-4,11,19,25,26,31,36]$. In this paper, we aim at optimal control problems under uncertainty, where the control objective involves a sparsifying term and, as a consequence, distributed optimal controls vanish on parts of the domain. The areas where controls are nonzero are interpreted as locations where it is most efficient to employ control devices [5,7,8,10,12,13,21,35].

Given a physical domain $\mathcal{D} \subset \mathbb{R}^{n}, n \in\{1,2,3\}$, we consider a partial differential equation involving uncertain parameters written as

$$
c(y, u, m(\omega))=0 .
$$

Here, $u$ and $y$ are the control and the state variables, respectively, $m(\omega)$ is an uncertain parameter, and $c(\cdot, \cdot, \cdot)$ denotes the PDE relating these variables. We assume that the distribution law of $m$, denoted by $\mu$, is supported on a Hilbert space $\mathscr{H}$, and consider $m$ as an $\mathscr{H}$-valued random variable. That is, for a probability space $(\Omega, \mathcal{F}, \mu)$, $m: \Omega \rightarrow \mathscr{H}$, where $\Omega$ is the set of events, $\mathcal{F}$ a $\sigma$-algebra of sets in $\Omega$, and $\mu$ is a positive normalized measure. For example, $\mathscr{H}$ can be chosen as infinite-dimensional

\footnotetext{
* Submitted to the editor November 20, 2018

Funding: Supported in part by the National Science Foundation under grants CBET-1507009 and DMS-1723211, and by the U.S. Department of Energy Office of Science, Advanced Scientific Computing Research (ASCR), Scientific Discovery through Advanced Computing (SciDAC) program.

†Courant Institute of Mathematical Sciences, New York University; lichen@cims.nyu.edu, stadler@cims.nyu.edu
} 
function space over $\mathcal{D}$ or its boundary $\partial \mathcal{D}$. We assume that for every $u \in L^{2}(\mathcal{D})$ and $\omega \in \Omega,(1)$ has a unique (weak) solution $y=y(u ; \omega, \cdot) \in V$, with an appropriate space $V \subset L^{2}(\mathcal{D}) .{ }^{1}$ For $\omega \in \Omega$, we consider the optimal control problem in reduced form,

$$
\min _{u \in U_{\mathrm{ad}}} J(\omega, u):=\frac{1}{2} \int_{\mathcal{D}}\left(y(u ; \omega, \cdot)-y_{d}\right)^{2} d \boldsymbol{x}+\frac{\alpha}{2} \int_{\mathcal{D}} u^{2} d \boldsymbol{x} .
$$

Here, $U_{\text {ad }}=\left\{u \in L^{2}(\mathcal{D}): a \leq u \leq b\right.$ a.e. $\}$, where $a, b \in L^{2}(\mathcal{D})$ with $a<b$ almost everywhere. Moreover, $\alpha>0$ is a regularization/control cost parameter and $y_{d} \in$ $L^{2}(\mathcal{D})$ a given desired state. For each $\omega \in \Omega,(2)$ is a classical control-constrained linear-quadratic optimal control problem. As is well known, this problem has a unique solution that depends on $\omega$.

We are interested in distributed optimal control problems, where the controls are sparse, i.e., they vanish on parts of the domain. We propose two practically relevant approaches to sparse optimal control under uncertainty. The first computes a deterministic sparse control that is optimal for the expectation of the cost functional. The second aims at stochastic controls that depend on the uncertain parameter, but have shared sparsity structure.

1.1. Deterministic sparse optimal control. Robust deterministic controls are optimal in expectation $[4,25,26]$, or optimal with respect to a risk measure $[1,27]$. Since in this formulation the controls are deterministic, it is straightforward to add a sparsity-enhancing term for the control to (2), resulting in

$$
\min _{u \in U_{\mathrm{ad}}} \mathcal{J}_{d}(u)=\frac{1}{2} \int_{\Omega} \int_{\mathcal{D}}\left(y(u ; \omega, \cdot)-y_{d}\right)^{2} d \boldsymbol{x} d \mu+\frac{\alpha}{2} \int_{\mathcal{D}} u^{2} d \boldsymbol{x}+\beta \int_{\mathcal{D}}|u| d \boldsymbol{x} .
$$

Here, $\beta>0$ is the weight for the sparsity-enhancing $L^{1}$-term, in which $|\cdot|$ denotes the absolute value. The deterministic optimal controls found from this formulation vanish on parts of the spatial domain $\mathcal{D}$, and the value of $\beta$ influences how sparse the control are. The resulting control structure can be used to decide on the placement of control devices. In the deterministic context, extensions of this approach have been applied for instance to optimal device placement in tissue imaging [5], sparse control of alignment models [7], optimal control of traveling wave fronts [10] or shaping controls for quantum systems [12].

1.2. Stochastic optimal control with shared sparsity. An alternative problems class and this paper's main focus is to find stochastic ${ }^{2}$ controls $u \in \boldsymbol{U}_{\mathrm{ad}}:=$ $\left\{u \in L_{\mu}^{2}\left(\Omega, L^{2}(\mathcal{D})\right), a \leq u(\omega, \cdot) \leq b\right.$ a.e. $\}$, i.e., individual controls $u=u(\omega)$ for each $\omega \in \Omega[3,11,31,36]$. These controls minimize the expected objective value, i.e.,

$$
\min _{u \in \boldsymbol{U}_{\mathrm{ad}}} \int_{\Omega} J(\omega, u(\omega, \cdot)) d \mu,
$$

with $J(\cdot, \cdot)$ as defined in (2). Note that (4) amounts to solving optimal control problems of the form (2) for each $\omega \in \Omega$, followed by computing the expectation over the values of the objective obtained with these optimal controls. One possibility for

\footnotetext{
${ }^{1}$ While the discussion is kept general in this introduction, in most of the remainder of this paper we focus on linear equations, where this assumption can easily be verified.

${ }^{2}$ While stochasticity is often used in the context of time-dependent problems, here it simply means that the controls depend on the random variable, in contrast to the deterministic optimal control formulation discussed above.
} 
incorporating sparsity in this formulation is to add sparsity-enhancing regularization for each $\omega$. However, since we interpret regions where optimal controls are non-zero as regions where we propose to place control devices, it is more meaningful to require that the stochastic controls share their sparsity structure. This can be achieved by adding a sparsity-enforcing term to the objective functional in (4):

$$
\min _{u \in \boldsymbol{U}_{\mathrm{ad}}} \mathcal{J}(u):=\int_{\Omega} J(\omega, u(\omega, \cdot)) d \mu+\beta \int_{\mathcal{D}}\left(\int_{\Omega}|u(\omega, \cdot)|^{2} d \mu\right)^{\frac{1}{2}} d \boldsymbol{x} .
$$

Here, $\beta>0$ and the outer integral in the sparsity-enforcing term is over the pointwise marginal distribution of the squared controls. Note that the sparsity term is well-defined and finite for $u \in \boldsymbol{U}_{\mathrm{ad}}$. As will be shown, using the $L^{1}$-norm of the pointwise expectation results in optimal controls $u(\omega, \cdot)$ with shared sparsity. While the optimal controls are stochastic, i.e., they depend on $\omega$, the controller locations resulting from (5) are deterministic, i.e., they only depend on the probability space, but not the individual event $\omega \in \Omega$. A practical interpretation of this approach is that the optimal location of controllers is computed by solving (5) in an offline phase, while the optimal controls $u(\omega)$ are computed in an online phase corresponding to the particular realization of the random variable $\omega$.

Let us give two application examples for an optimal control formulation of the form (21). First, we consider a problem from earthquake engineering, where one wants to find locations for active damping devices (controllers) that shall dampen vibrations that originate from an unknown earthquake forcing. In this situation, one can imagine that the optimal controls can be computed in real time individually for each forcing, but the location of controllers needs to be decided upfront and should be chosen in an optimal way for all possible earthquakes. Another application could be to position heaters in a building to obtain, for instance, a uniform temperature distribution in the presence of uncertain heat sinks/sources caused, e.g., by open windows, leaky walls or the presence of people.

1.3. Related work. Optimization under uncertainty governed by partial differential equations has been an active field of research over the last decade. Various formulations are proposed in the literature, e.g., robust deterministic optimal control $[1,19,25,27]$ and stochastic control $[3,11,36]$. The main focus of this paper is a stochastic control problem with linear governing equations, but with the additional requirement that the optimal controls are jointly sparse.

The interest in sparse optimal control is (1) due to its application for control device placement and its ability to discover controls with simple structure $[5,7,10$, $13,33,35]$, and (2) due to the interesting non-reflexive Banach space structure that arises if no Hilbert-space norm term is added to the objective $[8,9,13]$. The stochastic sparse control problem we study has similarities with the notion of directional sparsity proposed for optimal control of parabolic problems [21,28], in which one has to decide on the sparsity for an entire time stripe. However, differently from directional sparsity problems, our stochastic control formulation requires to decide on the sparsity based on a potentially high-dimensional integration over the probability space rather than an integration over the one-dimensional time direction.

The solution methods we propose are related to iteratively reweighted least squares (IRLS) algorithms, which are used, e.g., in compressive sensing, image processing and matrix recovery $[6,16,30]$. While IRLS methods have mostly been used in finite dimensions and in the context of underdetermined problems, they have recently also been studied for infinite-dimensional $L^{p}$ and $\ell^{p}(p \leq 1)$ optimization [23,24]. Instead 
of solving an $L^{p}$-problem directly, iterative reweighting algorithms alternate between solving a simpler (e.g., a quadratic minimization) problem, and updating a weighting function that enters into this simpler problem. In this paper we focus on a convex problem, but a significant challenge is that the optimization variable is defined over physical and infinite/high-dimensional random space. Hence, we employ ideas from iterative reweighting to avoid working with the high-dimensional optimization variable. The proposed algorithms only iterate over the reweighting function, which does not depend on the random variable. Additionally to the first-order IRLS method [16], we propose a Newton-type algorithm based on the reweighting formulation and show that it outperforms the classical IRLS iteration.

To accelerate norm reweighting methods, they have been combined with an active set method [24]. This approach also applies to nonconvex problems, but it requires the computation of norms of the optimization variable and the corresponding dual variable, similarly as for Newton-type methods for directional sparsity problems [21]. In the context of control under high-dimensional uncertainty, these norms are integrals over random space, making this integration a computational challenge.

Another attempt to accelerate the IRLS algorithm is to use the conjugate gradient method for solution of the auxiliary least squares problems that occurs in each iteration [18]. In section 5, we propose a related idea, but instead of iterative linear solves, we exploit the optimal control problem structure. Namely, we combine a low-rank operator approximation and the Sherman-Morrison-Woodbury identity to design fast (i.e., optimal complexity) iteration algorithms.

1.4. Contributions and limitations. The main contributions of this paper are as follows. (1) We propose a formulation for stochastic optimal controls with shared sparsity in the presence of uncertain parameters in the governing PDE. We believe that this formulation is relevant in applications and that it is interesting from the optimization-under-uncertainty perspective since the joint sparsity requirement couples the controls for different uncertain parameters, and from the sparse-control perspective due to the infinite-dimensional non-differentiable optimization structure. (2) We propose a Newton-type variant of the iteratively reweighted least squares (IRLS) minimization algorithm for the solution of this optimization problem, which is significantly faster than the classical IRLS method. (3) We present low-rank operator approximations which allow fast iterations of the IRLS algorithm and its Newton variant. We also provide bounds for the truncation error due to these approximations.

Next, we summarize limitations of this work. (1) We restrict ourselves to linear governing equations with uncertain parameters that enter linearly in these equations, and our algorithms for stochastic control with shared sparsity do not allow for control constraints. More general problems are a significant challenge even for optimal control without sparsity requirements on the controls. (2) The proposed norm reweighting problem reformulation requires regularization of the non-differentiable term in the objective and convergence to a truly sparse solution requires that the regularization parameter is driven to zero. (3) Our arguments and in particular the low-rank operator approximation require that the parameter $\alpha$ in (5) is positive. Using $\alpha=0$ does not allow a Hilbert space formulation and would be more challenging from the theoretical as well as the computational perspective.

1.5. Notation. We consider a probability space $(\Omega, \mathcal{F}, \mu)$, i.e., $\Omega$ is the set of events, $\mathcal{F}$ a $\sigma$-algebra of sets in $\Omega$, and $\mu$ a positive normalized measure. For $1 \leq$ $p<\infty$ and a Banach space $\left(X,\|\cdot\|_{X}\right)$, the Bochner space $L_{\mu}^{p}(\Omega ; X)$ is the space of 
Bochner integrable functions $u: \Omega \rightarrow X$, for which $\left(\int_{\Omega}\|u(\omega)\|_{X}^{p} d \mu\right)^{1 / p}:=\|u\|_{L^{p}(\Omega, X)}$ is finite. This value has the properties of a norm. For a domain $\mathcal{D} \subset \mathbb{R}^{d}, d \in\{1,2,3\}$, we will in particular use the spaces

$$
\boldsymbol{V}:=L_{\mu}^{2}\left(\Omega ; L^{2}(\mathcal{D})\right) \text { and } \boldsymbol{Y}:=L_{\mu}^{2}(\Omega ; Y),
$$

where $Y \subset H^{1}(\mathcal{D})$ is a subspace that can includes Dirichlet boundary conditions on part of $\partial \mathcal{D}$. Both, $\boldsymbol{V}$ and $\boldsymbol{Y}$ are Hilbert spaces with inner products derived from the inner products in $X$, e.g., the inner product for $\boldsymbol{V}$ is $\langle u, v\rangle_{V}:=\int_{\Omega}(u(\omega, \cdot), v(\omega, \cdot))_{L^{2}(\mathcal{D})} d \mu$ and the induced norm for $u \in \boldsymbol{V}$ is $\|u\|_{\boldsymbol{V}}=\langle u, u\rangle_{\boldsymbol{V}}^{1 / 2}$. For $u \in \boldsymbol{V}$, we will commonly use the notation

$$
\|u\|_{\Omega}=\|u\|_{\Omega}(\boldsymbol{x})=\left(\int_{\Omega} u(\omega, \boldsymbol{x})^{2} d \mu\right)^{1 / 2},
$$

which is well-defined due to the isomorphism between Bochner spaces and spaces defined over the product space $\Omega \times \mathcal{D}$ [22]. In the remainder of this paper, we use bold letters for function spaces defined over $\Omega \times \mathcal{D}$, such as $\boldsymbol{V}$ and $\boldsymbol{Y}$.

2. PDE with linearly-entering uncertain parameters. The main focus of this paper is on problems where the uncertain parameters enter linearly in (1). We allow for infinite-dimensional uncertain parameters that follow a Gaussian distribution $\mu=\mathcal{N}\left(m_{0}, \mathcal{C}_{0}\right)$ over a Hilbert space $\mathscr{H}$, where $m_{0} \in \mathscr{H}$, and $\mathcal{C}_{0}$ is a self-adjoint, positive definite trace class operator over $\mathscr{H}$. We consider $m$ as an $\mathscr{H}$-valued random variable and with a slight abuse of notation, we denote realization of this random variable by the same symbol $m$. We consider a linear differential equation of the form

$$
A y=u+f+B m,
$$

where $A: Y \subset H^{1}(\mathcal{D}) \mapsto H^{-1}(\mathcal{D})$ is invertible, $B: \mathscr{H} \mapsto H^{-1}(\mathcal{D})$ and $f, u \in H^{-1}(\mathcal{D})$. The following two examples fit into this framework.

Example 1 (Poisson problem with uncertain Robin boundary data). As example for an equation of the form (8), we use $\mathscr{H}=L^{2}\left(\partial \mathcal{D}_{2}\right)$ and consider a problem with inhomogeneous Robin boundary condition with uncertain data on $\partial \mathcal{D}_{2}$ :

$$
\begin{aligned}
-\nabla \cdot(a(\boldsymbol{x}) \nabla y(\omega, \boldsymbol{x})) & =f(\boldsymbol{x})+u(\boldsymbol{x}) & & \text { in } \mathcal{D}, \\
y(\omega, \boldsymbol{x}) & =0 & & \text { in } \partial \mathcal{D}_{1}, \\
k y(\omega, \boldsymbol{x})+(a(\boldsymbol{x}) \nabla y(\omega, \boldsymbol{x})) \cdot \boldsymbol{n} & =m(\omega) & & \text { in } \partial \mathcal{D}_{2} .
\end{aligned}
$$

Here, $a(\boldsymbol{x}) \geq a_{0}>0$ and $k \geq 0$. For $k=0$, the Robin condition on $\partial \mathcal{D}_{2}$ reduces to a Neumann boundary condition.

Example 2 (Poisson problem with uncertain right hand side). This example coincides with Example 1 with the exception that the uncertain parameter field enters on the right hand side of $(9 \mathrm{a})$. We use $\mathscr{H}=L^{2}(\tilde{\mathcal{D}})$ with $\tilde{\mathcal{D}} \subset \mathcal{D}$ an open subdomain, and $B: L^{2}(\tilde{\mathcal{D}}) \rightarrow L^{2}(\mathcal{D})$ is the extension-by-zero operator. The only difference to the problem above is that the term $B m(\omega)$ is added to the right hand side in (9a), whereas the right hand side of $(9 c)$ is zero.

Example 3 (Helmholtz problem with uncertain Neumann boundary data). Another example that fits into our framework is a Helmholtz problem with uncertain 
Neumann boundary forcing on $\partial \mathcal{D}_{2}$ :

$$
\begin{aligned}
-\Delta y(\omega, \boldsymbol{x})-\kappa^{2} y(\omega, \boldsymbol{x}) & =u(\boldsymbol{x}) & & \text { in } \mathcal{D}, \\
y(\omega, \boldsymbol{x}) & =0 & & \text { in } \partial \mathcal{D}_{1}, \\
\nabla y(\omega, \boldsymbol{x}) \cdot \boldsymbol{n} & =m(\omega) & & \text { in } \partial \mathcal{D}_{2} .
\end{aligned}
$$

Here, $\Delta$ is the Laplace operator and $\kappa>0$ is the wave number.

Most formulations and solution methods in this paper can straightforwardly be extended to generalizations of (8), e.g., to problems where $u$ is a vector function as in linear elasticity. However, for simplicity of the presentation, we restrict ourselves to (8). Next, we specialize the deterministic and stochastic optimal control formulations from subsection 1.1 and subsection 1.2 for this linear case.

2.1. Deterministic sparse optimal control. Using the linear equation (8), the deterministic sparse optimal control problem becomes

$$
\min _{u \in U_{\text {ad }}} \frac{1}{2} \int_{\Omega} \int_{\mathcal{D}}\left(A^{-1} u+A^{-1} B m-\hat{y}_{d}\right)^{2} d \boldsymbol{x} d \mu+\frac{\alpha}{2} \int_{\mathcal{D}} u^{2} d \boldsymbol{x}+\beta \int_{\mathcal{D}}|u| d \boldsymbol{x},
$$

where $\hat{y}_{d}=y_{d}-A^{-1} f$. Since the integration of a quadratic form over a Gaussian random variable can be done analytically [14, Remark 1.2.9], one obtains

$$
\begin{aligned}
\int_{\Omega} \int_{\mathcal{D}}\left(A^{-1} u\right. & \left.+A^{-1} B m-\hat{y}_{d}\right)^{2} d \boldsymbol{x} d \mu \\
= & \int_{\mathcal{D}}\left(A^{-1} u+A^{-1} B m_{0}-\hat{y}_{d}\right)^{2} d \boldsymbol{x}+\operatorname{Tr}\left(\mathcal{C}_{0}^{1 / 2} B^{\star} A^{-\star} A^{-1} B \mathcal{C}_{0}^{1 / 2}\right),
\end{aligned}
$$

where $B^{\star}$ denotes the adjoint of $B, A^{-\star}$ the adjoint of $A^{-1}$, and $\operatorname{Tr}(\cdot)$ denotes the trace of an operator. Note that since $\mathcal{C}_{0}$ is trace-class, and $B^{\star} A^{-\star} A^{-1} B$ is bounded on $L^{2}(\Omega)$, the trace in (12) is finite. Since the trace term does not depend on the control, it can be neglected in the computation of the minimizer. Thus, the optimal control derived from (11) is equivalently characterized by the minimization problem

$$
\min _{u \in U_{\mathrm{ad}}} \frac{1}{2} \int_{\mathcal{D}}\left(A^{-1} u-\tilde{y}_{d}\right)^{2} d \boldsymbol{x}+\frac{\alpha}{2} \int_{\mathcal{D}} u^{2} d \boldsymbol{x}+\beta \int_{\mathcal{D}}|u| d \boldsymbol{x},
$$

where $\tilde{y}_{d}=y_{d}-A^{-1} f+A^{-1} B m_{0}$. This is a deterministic elliptic control problem with $L^{1}$-control cost, where the desired state $\tilde{y}_{d}$ depends on the mean of the distribution of $m$. Problems of this form and algorithms for their solution have been studied for instance in [35], and have been generalized in various directions [8]. In particular, it is known that (13) admits a unique optimal control, which is sparse in the sense that it vanishes on parts of the domain $\mathcal{D}$. Moreover, this solution can be computed efficiently using a semismooth Newton algorithm in function space.

2.2. Stochastic optimal control with shared sparsity. Next, we consider the stochastic optimal control formulation (5) for the linear governing equation (8).

$$
\min _{u \in \boldsymbol{U}_{\mathrm{ad}}} \frac{1}{2} \int_{\Omega} \int_{\mathcal{D}}\left(\left(A^{-1} u+A^{-1} B m-\hat{y}_{d}\right)^{2}+\alpha u^{2}\right) d \boldsymbol{x} d \mu+\beta \int_{\mathcal{D}}\left(\int_{\Omega} u(\omega, \cdot)^{2} d \mu\right)^{1 / 2} d \boldsymbol{x}
$$

where, as above $\hat{y}_{d}=y_{d}-A^{-1} f$. The added sparsity term is an infinite-dimensional version of an $\ell^{2,1}$-norm, used in finite dimensions to achieve group sparsity structure 
or matrix sparsity [29,32]. In general, for $r, p>0$, the $\ell^{r, p}$-norm of a two-index array $a_{i, j}, 1 \leq i \leq n, 1 \leq j \leq m$ is defined as

$$
\|a\|_{r, p}:=\left[\sum_{i=1}^{n}\left(\sum_{j=1}^{m}\left|a_{i, j}\right|^{r}\right)^{\frac{p}{r}}\right]^{\frac{1}{p}} .
$$

In (14), we aim at obtaining shared sparsity structure amongst controls for different uncertain parameters. If the integrand vanishes at a point $\boldsymbol{x} \in \mathcal{D}$, then the controls for almost all random parameters must vanish at this $\boldsymbol{x}$, resulting in shared sparsity. We also notice that the sparsifying term couples the problems for different random variables $\omega$ and thus one cannot integrate over the random space analytically. The properties and solution algorithms for (14) are the main focus of this paper. Note that this problem is related to the directional sparsity formulation for time-dependent optimal control proposed in [21], with the stochastic space taking the role of the time direction. One of the main differences between the directional sparsity and stochastic control with shared sparsity is the high dimension of the probability space compared to the one-dimensional time variable. Due to this difference, the generalized Newton algorithms used to solve directional sparsity control problems cannot be applied for the solution of (14). In the next section, we characterize solutions to the shared sparsity control problem and introduce a regularized variant of (14).

3. Properties of shared sparsity stochastic control problem. We first introduce the notation $Q: U_{\text {ad }} \times \mathscr{H} \rightarrow \mathbb{R}$,

$$
Q(u, m):=\frac{1}{2} \int_{\Omega} \int_{\mathcal{D}}\left(\left(A^{-1} u+A^{-1} B m-\hat{y}_{d}\right)^{2}+\alpha u^{2}\right) d \boldsymbol{x} d \mu .
$$

First, we summarize necessary and sufficient optimality conditions for solutions to (14) using the notation introduced in (7).

THEOREM 4. The optimal control problem (14) has a unique solution $\bar{u} \in \boldsymbol{U}_{a d}$, characterized by the existence of corresponding state $\bar{y} \in \boldsymbol{Y}$, adjoint state $\bar{p} \in \boldsymbol{Y}$ and multiplier $\bar{\lambda} \in \boldsymbol{V}$ such that

$$
\begin{aligned}
& A \bar{y}-\bar{u}-f-B m=0, \\
& A^{\star} \bar{p}-y_{d}+\bar{y}=0, \\
& -\bar{p}+\alpha \bar{u}+\beta \bar{\lambda}+\bar{\mu}=0, \\
& \left.\begin{array}{ll}
\bar{\lambda}(\omega, \boldsymbol{x})=\frac{\bar{u}(\omega, \boldsymbol{x})}{\|\bar{u}\|_{\Omega}(\boldsymbol{x})} & \text { for } \boldsymbol{x} \in \mathcal{D} \text { with }\|\bar{u}\|_{\Omega}(\boldsymbol{x}) \neq 0 \\
\|\bar{\lambda}\|_{\Omega}(\boldsymbol{x}) \leq 1 & \text { for } \boldsymbol{x} \in \mathcal{D} \text { with }\|\bar{u}\|_{\Omega}(\boldsymbol{x})=0
\end{array}\right\} \text { for a.a. } \boldsymbol{x} \in \mathcal{D}, \\
& \bar{\mu} \leq 0 \text { if } \bar{u}=a, \quad \bar{\mu} \geq 0 \text { if } \bar{u}=b, \text { and } \bar{\mu}=0 \text { if } a \leq \bar{u} \leq b \text { a.e. in } \mathcal{D} \times \Omega \text {. }
\end{aligned}
$$

Proof. We denote the sparsifying term in (14) by $\varphi(u)$, i.e., $\varphi(u):=\int_{\mathcal{D}}\|u\|_{\Omega} d \boldsymbol{x}$. It follows from convex analysis [17] that the variational inequality

$$
\left\langle Q_{u}(\bar{u}, m), u-\bar{u}\right\rangle_{\boldsymbol{V}}+\beta(\varphi(u)-\varphi(\bar{u})) \geq 0 \quad \text { for all } u \in \boldsymbol{U}_{\mathrm{ad}},
$$

is necessary and sufficient for $\bar{u}$ to be a solution of (14), where $Q_{u}$ denotes variation of $Q$ with respect to $u$. This is equivalent to

$$
\left\langle A^{-\star}\left(A^{-1} \bar{u}+A^{-1} B m-\hat{y}_{d}\right)+\alpha \bar{u}+\beta \bar{\lambda}, u-\bar{u}\right\rangle_{\boldsymbol{V}} \geq 0 \quad \text { for all } u \in \boldsymbol{U}_{\mathrm{ad}},
$$


where $\bar{\lambda} \in \boldsymbol{V}$ is an element in the subdifferential $\partial \varphi(\bar{u})$ of $\varphi$ at $\bar{u}$. By introducing the adjoint variable $\bar{p}$ and a Lagrange multiplier $\bar{\mu} \in \boldsymbol{V}$ associated with the bound constraints in $\boldsymbol{U}_{\text {ad }}$, (17) results in (15a)-(15c) and (15e).

It remains to show that $\bar{\lambda} \in \partial \varphi(\bar{u})$ is equivalent with (15d). Considering $\varphi: \boldsymbol{V} \rightarrow$ $\mathbb{R}$, the subdifferential is defined as

$$
\partial \varphi(\bar{u})=\left\{\lambda \in \boldsymbol{V} \mid\langle\lambda, v-\bar{u}\rangle_{\boldsymbol{V}} \leq \int_{\mathcal{D}}\left(\|v\|_{\Omega}-\|\bar{u}\|_{\Omega}\right) d \boldsymbol{x} \text { for any } v \in \boldsymbol{V}\right\} .
$$

To show equivalence, let us first assume that $\bar{\lambda} \in \partial \varphi(\bar{u})$. Choosing $v:=\bar{u}+\bar{\lambda} \delta$, where $\delta \in L^{\infty}(\mathcal{D})$, i.e., it only depends on $\boldsymbol{x}$, we obtain: $\int_{\mathcal{D}} \delta\|\bar{\lambda}\|_{\Omega}^{2} d \boldsymbol{x}=\langle\bar{\lambda}, \delta \bar{\lambda}\rangle_{\boldsymbol{V}} \leq$ $\int_{\mathcal{D}} \delta\|\bar{\lambda}\|_{\Omega} d \boldsymbol{x}$, which implies that $\|\bar{\lambda}\|_{\Omega} \leq 1$. Setting $v=0$ in (18) shows that

$$
\int_{\mathcal{D}}\|\bar{u}\|_{\Omega} d \boldsymbol{x} \leq\langle\lambda, \bar{u}\rangle_{\boldsymbol{V}} \leq \int_{\mathcal{D}}\|\bar{\lambda}\|_{\Omega}\|\bar{u}\|_{\Omega} d \boldsymbol{x} \leq \int_{\mathcal{D}}\|\bar{u}\|_{\Omega} d \boldsymbol{x}
$$

where we have used Hölder's inequality for the second estimate. For $\boldsymbol{x} \in \mathcal{D}$ with $\|\bar{u}\|_{\Omega}(\boldsymbol{x}) \neq 0$, thus necessarily $\bar{\lambda}(\omega, \boldsymbol{x})=\bar{u}(\omega, \boldsymbol{x}) /\|\bar{u}\|_{\Omega}(\boldsymbol{x})$. Thus, we have shown that $\bar{\lambda} \in \partial \varphi(\bar{u})$ implies that $(\bar{u}, \bar{\lambda})$ satisfies $(15 \mathrm{~d})$. Conversely, we assume that $\bar{u}$ and $\bar{\lambda}$ satisfy (15d) and we split $\mathcal{D}$ into $\mathcal{D}_{1}=\left\{\boldsymbol{x} \in \mathcal{D} \mid\|\bar{u}\|_{\Omega}(\boldsymbol{x})=0\right\}$ and $\mathcal{D}_{2}=\mathcal{D} \backslash \mathcal{D}_{1}$. For any $v \in \boldsymbol{V}$, we then have on $\mathcal{D}_{1}$,

$$
\int_{\mathcal{D}_{1}} \int_{\Omega} \bar{\lambda} v d \mu d \boldsymbol{x} \leq \int_{\mathcal{D}_{1}}\|\bar{\lambda}\|_{\Omega}\|v\|_{\Omega} d \boldsymbol{x} \leq \int_{\mathcal{D}_{1}}\|v\|_{\Omega} d \boldsymbol{x}=\int_{\mathcal{D}_{1}}\|v\|_{\Omega}-\|\bar{u}\|_{\Omega} d \boldsymbol{x}
$$

and on $\mathcal{D}_{2}$,

$$
\int_{\mathcal{D}_{2}} \int_{\Omega} \bar{\lambda}(v-\bar{u}) d \mu d \boldsymbol{x}=\int_{\mathcal{D}_{2}} \int_{\Omega} \frac{\bar{u}}{\|\bar{u}\|_{\Omega}}(v-\bar{u}) d \mu d \boldsymbol{x} \leq \int_{\mathcal{D}_{2}}\|v\|_{\Omega}-\|\bar{u}\|_{\Omega} d \boldsymbol{x},
$$

where we have used the Cauchy-Schwartz inequality. Combining (19) and (20), we find that $\bar{\lambda} \in \partial \varphi(\bar{u})$, which ends the proof.

We now define the following family of regularized control objectives for $\varepsilon \geq 0$.

$$
\mathcal{J}(u, \varepsilon):=Q(u, m)+\beta \int_{\mathcal{D}}\left(\|u\|_{\Omega}^{2}+\varepsilon^{2}\right)^{1 / 2} d \boldsymbol{x} .
$$

In particular, the objective in (14) is $\mathcal{J}(u, 0)$. A result similar to Theorem 4 also holds for the regularized problem (21), where the objective function is now differentiable.

COROLlaRY 5. The regularized problem (21) with $\varepsilon>0$ has a unique solution $u_{\varepsilon} \in \boldsymbol{U}_{a d}$, characterized by the existence of corresponding state $y_{\varepsilon} \in \boldsymbol{Y}$, adjoint $p_{\varepsilon} \in \boldsymbol{Y}$ and multiplier $\lambda_{\varepsilon} \in \boldsymbol{V}$ such that

$$
\begin{gathered}
A y_{\varepsilon}-u_{\varepsilon}-f-B m=0 \\
A^{\star} p_{\varepsilon}-y_{d}+y_{\varepsilon}=0 \\
-p_{\varepsilon}+\alpha u_{\varepsilon}+\beta \frac{u_{\varepsilon}}{\sqrt{\left\|u_{\varepsilon}\right\|_{\Omega}^{2}+\varepsilon^{2}}}+\mu_{\varepsilon}=0 \text { for a.a. } \boldsymbol{x} \in \mathcal{D} \\
\mu_{\varepsilon} \leq 0 \text { if } u_{\varepsilon}=a, \mu_{\varepsilon} \geq 0 \text { if } u_{\varepsilon}=b, \text { and } \mu_{\varepsilon}=0 \text { if } a \leq u_{\varepsilon} \leq b \text { a.e. in } \mathcal{D} \times \Omega .
\end{gathered}
$$

The next result provides a bound for the difference between minimizers of (14) and its $\varepsilon$-regularized version with objective (21). 
Lemma 6. Let $\mathcal{D}$ be bounded, $\varepsilon>0$ and denote by $\bar{u}$ the solution to (14), i.e., the minimizer of $u \mapsto \mathcal{J}(u, 0)$, and by $u_{\varepsilon}$ the minimizer of $u \mapsto \mathcal{J}(u, \varepsilon)$. Then

$$
\left\|\bar{u}-u_{\varepsilon}\right\|_{\boldsymbol{V}}^{2} \leq \varepsilon \beta \alpha^{-1}|\mathcal{D}|,
$$

where $|\mathcal{D}|$ denotes the volume of $\mathcal{D}$.

Proof. Since $\bar{u}$ and $u_{\varepsilon}$ are the unique minimizers of (21) for $\varepsilon=0$ and $\varepsilon>0$, respectively, we have for all $v \in \boldsymbol{U}_{\text {ad }}$ that

$$
\left\langle\left(A^{-\star} A^{-1}+\alpha\right) u_{\varepsilon}, v-u_{\varepsilon}\right\rangle_{\boldsymbol{V}}+\beta \int_{D}\left(\sqrt{\|v\|_{\Omega}^{2}+\varepsilon^{2}}-\sqrt{\left\|u_{\varepsilon}\right\|_{\Omega}^{2}+\varepsilon^{2}}\right) d \boldsymbol{x} \geq-\left\langle g, v-u_{\varepsilon}\right\rangle_{\boldsymbol{V}},
$$

$$
\left\langle\left(A^{-\star} A^{-1}+\alpha\right) \bar{u}, v-\bar{u}\right\rangle_{\boldsymbol{V}}+\beta \int_{D}\left(\|v\|_{\Omega}-\|\bar{u}\|_{\Omega}\right) d \boldsymbol{x} \geq-\langle g, v-\bar{u}\rangle_{\boldsymbol{V}},
$$

where $g=A^{-\star}\left(A^{-1} B m-\hat{y}_{d}\right)$. Using $v=u_{\varepsilon}$ in (24) and $v=\bar{u}$ in (25), and summing the resulting inequalities yields

$$
\begin{aligned}
& \left\langle\left(A^{-\star} A^{-1}+\alpha\right)\left(u_{\varepsilon}-\bar{u}\right), u_{\varepsilon}-\bar{u}\right\rangle_{\boldsymbol{V}} \leq \\
& \quad \beta \int_{D}\left(\left\|u_{\varepsilon}\right\|_{\Omega}-\|\bar{u}\|_{\Omega}+\sqrt{\|\bar{u}\|_{\Omega}^{2}+\varepsilon^{2}}-\sqrt{\left\|u_{\varepsilon}\right\|_{\Omega}^{2}+\varepsilon^{2}}\right) d \boldsymbol{x} .
\end{aligned}
$$

The expression under the integral on the right hand side can be estimated pointwise:

$$
\begin{aligned}
\left\|u_{\varepsilon}\right\|_{\Omega}-\sqrt{\left\|u_{\varepsilon}\right\|_{\Omega}^{2}+\varepsilon^{2}}-\|\bar{u}\|_{\Omega}+\sqrt{\|\bar{u}\|_{\Omega}^{2}+\varepsilon^{2}} & \leq \sqrt{\|\bar{u}\|_{\Omega}^{2}+\varepsilon^{2}}-\|\bar{u}\|_{\Omega} \\
& \leq \frac{\varepsilon^{2}}{\sqrt{\|\bar{u}\|_{\Omega}^{2}+\varepsilon^{2}}+\|\bar{u}\|_{\Omega}} \leq \varepsilon .
\end{aligned}
$$

Integrating this estimate over $\mathcal{D}$ and combination with (26) proves the result.

In the next two sections, we introduce a first and a second-order algorithm for the solution of (21) without bound constraints on the control. Both algorithms avoid approximation in random space using sampling and only iterate over functions defined on the physical space $\mathcal{D}$. Thanks to the linearity of the governing equation and the Gaussianity of the uncertain parameter, computations over the (potentially high-dimensional) random space $\Omega$ can be performed analytically. In practice, these computations can be performed efficiently using low-rank operator approximations as proposed in section 6 .

4. Norm reweighting for shared sparsity control problem. To develop an efficient algorithm for (14), we make the simplification $\boldsymbol{U}_{\mathrm{ad}}=\boldsymbol{V}=L_{\mu}^{2}\left(\Omega ; L^{2}(\mathcal{D})\right.$ ), i.e., we consider a problem without bound constraints on the control. The algorithm we propose below cannot be generalized to incorporate inequality constraints. Such constraints would destroy the Gaussianity of the controls in the auxiliary problems we introduce below. We introduce a family of objective functions that are quadratic in $u$, involve the parameter $\varepsilon \geq 0$ and a weighting function $\nu: \mathcal{D} \rightarrow \mathbb{R}$ with $\nu(\boldsymbol{x})>0$ :

$$
\overline{\mathcal{J}}(u, \nu, \varepsilon):=Q(u, m)+\frac{\beta}{2} \int_{\mathcal{D}}\left(\nu\|u\|_{\Omega}^{2}+\varepsilon^{2} \nu+\nu^{-1}\right) d \boldsymbol{x} .
$$

Here, the function $\nu$ weights the term $\|u\|_{\Omega}^{2}(\boldsymbol{x})$, and the latter two terms, which are not present in (14) will be useful to update the weighting function $\nu$ in the algorithm 
presented next. Norm reweighting, in the context of under-determined problems also known as iteratively reweighted least squares (IRLS), is commonly used to compute finite-dimensional sparse solutions vectors. Some of the analysis presented below extends results from [16] to infinite dimensions. Similar generalizations to infinite dimensions, also for the non-convex case, are presented in [23,24], where the method is referred to as monotone algorithm. The basic idea behind these methods applied to the stochastic shared sparsity control problem is presented next.

For a given, monotonously decreasing sequence $\left(\varepsilon_{k}\right)_{k \geq 0}$ with $\varepsilon_{k}>0$, the algorithm performs alternate minimization of $\overline{\mathcal{J}}(u, \nu, \varepsilon)$ with respect to $u$ and $\nu$. Given an initialization $\nu^{0}, \nu_{0}(\boldsymbol{x})>0$ for all $\boldsymbol{x} \in \mathcal{D}$, we compute, for $k \geq 1$, a sequence of iterates $u^{k}=u^{k}(\omega, \boldsymbol{x}), \nu^{k}(\boldsymbol{x})$ as follows:

$$
\begin{aligned}
& u^{k+1}=\underset{u \in \boldsymbol{V}}{\arg \min } \overline{\mathcal{J}}\left(u, \nu^{k}, \varepsilon_{k}\right), \\
& \nu^{k+1}=\underset{\nu \in L^{\infty}(\mathcal{D})}{\arg \min } \overline{\mathcal{J}}\left(u^{k+1}, \nu, \varepsilon_{k+1}\right) .
\end{aligned}
$$

Let us first discuss the minimization (29). Taking variations of (27) with respect to $\nu$, and using that $\nu$ must be positive, (29) implies that

$$
\nu^{k+1}(\boldsymbol{x})=\left(\left\|u^{k+1}\right\|_{\Omega}^{2}(\boldsymbol{x})+\varepsilon_{k+1}^{2}\right)^{-\frac{1}{2}} .
$$

Note that $\nu^{k+1}$ is a function only of $\boldsymbol{x} \in \mathcal{D}$, and, for each $\boldsymbol{x}$, it requires integration over the random space $\Omega$.

Since (28) is a strictly convex least squares ${ }^{3}$ problem, it has a unique solution $u^{k+1}$. Taking variations with respect to $u$, one finds that $u^{k+1}$ is characterized by the optimality condition

$$
\left[A^{-\star} A^{-1}+\left(\alpha+\beta \nu^{k}\right)\right] u^{k+1}=A^{-\star}\left(y_{d}-A^{-1}(f+B m)\right) .
$$

After introducing state and adjoint variables $y^{k+1}, p^{k+1} \in \boldsymbol{Y}$, this is equivalent to

$$
\begin{aligned}
A y^{k+1}-u^{k+1}-f-B m & =0, \\
A^{\star} p^{k+1}-y_{d}+y^{k+1} & =0, \\
-p^{k+1}+\left(\alpha+\beta \nu^{k}\right) u^{k+1} & =0 .
\end{aligned}
$$

Next, we observe that using the optimality condition (30) in (27) yields

$$
\overline{\mathcal{J}}\left(u^{k}, \nu^{k}, \varepsilon_{k}\right)=Q\left(u^{k}, m\right)+\beta \int_{\mathcal{D}}\left(\left\|u^{k}\right\|_{\Omega}^{2}+\varepsilon_{k}^{2}\right)^{1 / 2} d \boldsymbol{x}=\mathcal{J}\left(u^{k}, \varepsilon_{k}\right),
$$

shining light onto the relation between $\mathcal{J}$ and $\overline{\mathcal{J}}$. Note, however, that $u^{k}$ is in general not a minimizer of $u \mapsto \mathcal{J}\left(u, \varepsilon_{k}\right)$.

The alternate minimization property (28), (29) shows that the following monotonicity holds for $k=0,1,2, \ldots$,

$$
\overline{\mathcal{J}}\left(u^{k+1}, \nu^{k+1}, \varepsilon_{k+1}\right) \leq \overline{\mathcal{J}}\left(u^{k+1}, \nu^{k}, \varepsilon_{k+1}\right) \leq \overline{\mathcal{J}}\left(u^{k+1}, \nu^{k}, \varepsilon_{k}\right) \leq \overline{\mathcal{J}}\left(u^{k}, \nu^{k}, \varepsilon_{k}\right) .
$$

Here, the first inequality follows from the optimality of $\nu^{k+1}$ for (29), the second inequality from the definition (21) and from $\varepsilon_{k+1} \leq \varepsilon_{k}$, and the last inequality from

\footnotetext{
${ }^{3}$ The occurrence of this least square problem is the reason why this algorithm is referred to as iteratively reweighted least squares (IRLS) method in the literature [16].
} 
the optimality of $u^{k+1}$ for (28). ${ }^{4}$ The iterates of the algorithm satisfy a boundedness property summarized in the next lemma.

LEMMA 7. Let $\varepsilon_{k}, k=0,1, \ldots$ be a non-increasing sequence of positive numbers, and $\left(u^{0}, \nu^{0}\right)$ a given initialization. Then, the iterates $u^{k}$ satisfy

$$
\sum_{k=0}^{\infty} \int_{\Omega} \int_{\mathcal{D}}\left(u^{k}-u^{k+1}\right)^{2} d \boldsymbol{x} d \mu<\infty
$$

Proof. The following estimate holds:

$$
\begin{aligned}
& \overline{\mathcal{J}}\left(u^{k}, \nu^{k}, \varepsilon_{k}\right)-\overline{\mathcal{J}}\left(u^{k+1}, \nu^{k+1}, \varepsilon_{k+1}\right) \\
\geq & \overline{\mathcal{J}}\left(u^{k}, \nu^{k}, \varepsilon_{k}\right)-\overline{\mathcal{J}}\left(u^{k+1}, \nu^{k}, \varepsilon_{k}\right) \\
\geq & \frac{1}{2} \int_{\Omega} \int_{\mathcal{D}}\left(A^{-1}\left(u^{k}-u^{k+1}\right)\right)^{2}+\left(\alpha+\beta \nu^{k}\right)\left(u^{k}-u^{k+1}\right)^{2} d \boldsymbol{x} d \mu \\
\geq & \frac{\alpha}{2} \int_{\Omega} \int_{\mathcal{D}}\left(u^{k}-u^{k+1}\right)^{2} d \boldsymbol{x} d \mu .
\end{aligned}
$$

Here, the first inequality uses (33), and the second inequality follows from a Taylor expansion of $u \mapsto \overline{\mathcal{J}}\left(u, \nu^{k}, \varepsilon_{k}\right)$ at $u^{k+1}$ in the direction $u^{k}-u^{k+1}$, in which due to (28) the first-order term vanishes, i.e. $\left\langle u^{k+1}, u^{k}-u^{k+1}\right\rangle_{\boldsymbol{V}}=0$. Summing the above estimate over $k$ proves (34).

Note that the above result implies that, in particular,

$$
\int_{\Omega} \int_{\mathcal{D}}\left(u^{k}-u^{k+1}\right)^{2} d \boldsymbol{x} d \mu \rightarrow 0 \quad \text { as } k \rightarrow \infty .
$$

However, Lemma 7 does not imply convergence of the IRLS algorithm when $\varepsilon_{k} \rightarrow 0$. The next result provides a convergence result for the case that $\varepsilon_{k} \rightarrow \bar{\varepsilon}>0$.

Lemma 8. Let $\varepsilon_{k}, k=1,2, \ldots$ be a non-increasing sequence with $\lim _{k \rightarrow \infty} \varepsilon_{k}=$ $\bar{\varepsilon}>0$. Then, for any initialization $\left(u^{0}, \nu^{0}\right), u^{k} \rightarrow u_{\bar{\varepsilon}}$ strongly in $\boldsymbol{V}$ as $k \rightarrow \infty$.

Proof. As above, we denote by $p^{k} \in \boldsymbol{V}$ the adjoint variable corresponding to $u^{k}$. We consider the derivative of $\mathcal{J}$ with respect to $u, \mathcal{J}_{u}\left(u^{k}, \bar{\varepsilon}\right)=-p^{k}+\alpha u^{k}+\beta \bar{\nu}^{k} u^{k}$, where $\bar{\nu}^{k}=\left(\left\|u^{k}\right\|_{\Omega}^{2}+\bar{\varepsilon}^{2}\right)^{-1 / 2}$. Then,

$$
\begin{array}{r}
\left\|-p^{k}+\alpha u^{k}+\beta \bar{\nu}^{k} u^{k}\right\|_{\boldsymbol{V}}=\left\|-\left(p^{k}-p^{k+1}\right)+\alpha\left(u^{k}-u^{k+1}\right)+\beta\left(\bar{\nu}^{k} u^{k}-\nu^{k} u^{k+1}\right)\right\|_{\boldsymbol{V}} \\
\leq\left\|p^{k}-p^{k+1}\right\|_{\boldsymbol{V}}+\alpha\left\|u^{k}-u^{k+1}\right\|_{\boldsymbol{V}}+\beta \bar{\varepsilon}^{-1}\left\|u^{k}-u^{k+1}\right\|_{\boldsymbol{V}} \\
+\beta \bar{\varepsilon}^{-2}\left\|u^{k+1}\left(\sqrt{\left\|u^{k}\right\|_{\Omega}^{2}+\bar{\varepsilon}^{2}}-\sqrt{\left\|u^{k}\right\|_{\Omega}^{2}+\varepsilon_{k}^{2}}\right)\right\|_{\boldsymbol{V}}
\end{array}
$$

where we used that $-p^{k+1}+\alpha u^{k+1}+\nu^{k} u^{k+1}=0$ in the first equality, with $u^{k+1}$ is the minimizer of $\overline{\mathcal{J}}\left(u, \nu^{k}, \epsilon_{k}\right), \nu^{k}$ defined as (30) and the assumption $\varepsilon_{k} \geq \bar{\varepsilon}$ in the estimation. Using Lemma 7 and the fact that $\varepsilon_{k} \rightarrow \bar{\varepsilon}$ implies that $J_{u}\left(u^{k}, \bar{\varepsilon}\right) \rightarrow 0$ as $k \rightarrow \infty$. Finally, since $\left\|J_{u}\left(u^{k}, \bar{\varepsilon}\right)\right\|_{\boldsymbol{V}} \geq \alpha\left\|u^{k}-u_{\bar{\varepsilon}}\right\|_{\boldsymbol{V}}$, we obtain the postulated convergence result.

\footnotetext{
${ }^{4}$ This monotonicity property of the algorithm is the reason why this class of algorithms are referred to as monotone algorithms in [23,24].
} 
5. Newton method for reweighted shared sparsity control problem. Rather than using optimization objectives that depend on the control $u \in \boldsymbol{V}$, or both on the control $u$ and the weighting function $\nu$, here we propose a reduced objective that only depends on $\nu$ (and on $\varepsilon$ ). This objective considers $u$ as a function of $\nu$ and thus a numerical scheme for this reduced formulation only requires iterations for $\nu$. The optimality condition with respect to $u$ in (27) shows that

$$
u=S_{\nu} v,
$$

where $S_{\nu}: \boldsymbol{V} \rightarrow \boldsymbol{V}$ and $v$ are defined as

$$
S_{\nu}:=\left[A^{-\star} A^{-1}+(\alpha+\beta \nu)\right]^{-1}, \quad v:=A^{-\star}\left(y_{d}-A^{-1}(f+B m)\right) .
$$

Since for every $\nu \in L^{\infty}(\mathcal{D}), \nu \geq 0$, (35) has a unique solution $u$, we can consider $u$ as a function of $\nu$ only, leading to the following reduced version of (27),

$$
\tilde{\mathcal{J}}(\nu, \varepsilon):=Q\left(S_{\nu} v, m\right)+\frac{\beta}{2} \int_{\mathcal{D}}\left(\nu\left\|S_{\nu} v\right\|_{\Omega}^{2}+\varepsilon^{2} \nu+\nu^{-1}\right) d \boldsymbol{x} .
$$

Note that this is a non-quadratic functional in $\nu$. Its derivative in a direction $\tilde{\nu}$ is:

$$
\begin{aligned}
\tilde{\mathcal{J}}_{\nu}(\nu, \varepsilon)(\tilde{\nu})= & \int_{\mathcal{D}}\left(A^{-\star}\left(A^{-1} S_{\nu} v+A^{-1} B m-\hat{y}_{d}\right)+\alpha S_{\nu} v+\beta \nu S_{\nu} v\right)\left(S_{\nu} v\right)_{, \nu}(\tilde{\nu}) d \boldsymbol{x} \\
& +\frac{\beta}{2} \int_{\mathcal{D}}\left(\left\|S_{\nu} v\right\|_{\Omega}^{2}+\varepsilon^{2}-\frac{1}{\nu^{2}}\right) \tilde{\nu} d \boldsymbol{x} \\
= & \frac{\beta}{2} \int_{\mathcal{D}}\left(\left\|S_{\nu} v\right\|_{\Omega}^{2}+\varepsilon^{2}-\frac{1}{\nu^{2}}\right) \tilde{\nu} d \boldsymbol{x},
\end{aligned}
$$

where $\left(S_{\nu} v\right)_{, \nu}$ denotes variation of $S_{\nu} v$ with respect to $\nu$, and the first term in the second expression vanishes since $\left[A^{-\star} A^{-1}+(\alpha+\beta \nu)\right] S_{\nu} v-A^{-\star}\left(\hat{y}_{d}-A^{-1} B m\right)=0$. Using the $L^{2}(\mathcal{D})$-inner product, the gradient $\mathcal{G}$ of $\tilde{\mathcal{J}}$ with respect to $\nu$ is thus

$$
\mathcal{G}(\nu)=\left\|S_{\nu} v\right\|_{\Omega}^{2}+\varepsilon^{2}-\frac{1}{\nu^{2}},
$$

where for simplicity, we neglect to denote the dependence of $\mathcal{G}$ on $\varepsilon$. Note that using (35) and (36), and introducing the control, state and adjoint variables $u_{\varepsilon}, y_{\varepsilon}$ and $p_{\varepsilon}$, the first-order optimality condition $\mathcal{G}(\nu)=0$ is equivalent to the optimality system (22a), (22b), (22c) with $\mu_{\varepsilon}=0$. This system uniquely characterizes $u_{\varepsilon}$, and thus the corresponding $\nu_{\varepsilon}=\left(\left\|u_{\varepsilon}\right\|_{\Omega}^{2}+\varepsilon^{2}\right)^{1 / 2}$ satisfies $\mathcal{G}\left(\nu_{\varepsilon}\right)=0$ and is thus the unique minimizer of (37).

A possible choice for an iterative fixed-point method to solve $\mathcal{G}(\nu)=0$ with $\varepsilon=\varepsilon_{k+1}>0$ is, for given $\nu^{k}$, to compute $\nu^{k+1}$ from

$$
\frac{1}{\left(\nu^{k+1}\right)^{2}}=\left\|S_{\nu^{k}} v\right\|_{\Omega}^{2}+\varepsilon_{k+1}^{2}
$$

Taking square roots and reciprocals, we thus rediscover the IRLS method from section 4 as an iterative fixed point method for solving $\mathcal{G}(\nu)=0$. This also implies that the gradient can be computed from the iterates of the IRLS algorithm as

$$
\mathcal{G}\left(\nu^{k}\right)=\frac{1}{\left(\nu^{k+1}\right)^{2}}-\frac{1}{\left(\nu^{k}\right)^{2}},
$$


which provides a possible termination criterion for the IRLS algorithm.

Computing second variations of $\tilde{\mathcal{J}}$ with respect to $\nu$ in a direction $\tilde{\nu}$ yields the following Hessian operator

$$
\mathcal{H}(\nu) \tilde{\nu}=-2 \beta \int_{\Omega}\left(S_{\nu} v\right) \odot S_{\nu}\left(\left(S_{\nu} v\right) \odot \tilde{\nu}\right) d \mu+\frac{2}{\nu^{3}} \odot \tilde{\nu},
$$

where $v$ as defined in (36) is a Gaussian random process, and $\odot$ denotes the pointwise multiplication in space. That is, for $f, g: \mathcal{D} \rightarrow \mathbb{R}$ and $h: \mathcal{D} \times \Omega \rightarrow \mathbb{R},(f \odot g)(\boldsymbol{x}):=$ $f(\boldsymbol{x}) g(\boldsymbol{x})$, and $(h \odot g)(\boldsymbol{x}, \omega):=h(\boldsymbol{x}, \omega) g(\boldsymbol{x})$. In the derivation of (41), we have also used that the derivative of $S_{\nu} v$ with respect to $\nu$ satisfies $\left(S_{\nu} v\right)_{, \nu} \tilde{\nu}=-\beta S_{\nu}\left(\left(S_{\nu} v\right) \odot \tilde{\nu}\right)$ for any random draw $v(\omega)$. Thus, the Newton update step at an iterate $\nu=\nu^{k}$ is

$$
\begin{gathered}
-2 \beta \int_{\Omega}\left(S_{\nu^{k}} v\right) \odot S_{\nu^{k}}\left(\left(S_{\nu^{k}} v\right) \odot \delta \nu\right) d \mu+\frac{2}{\left(\nu^{k}\right)^{3}} \odot \delta \nu=-\left\|S_{\nu^{k}} v\right\|_{\Omega}^{2}-\varepsilon_{k+1}^{2}+\frac{1}{\left(\nu^{k}\right)^{2}} \\
\nu^{k+1}=\nu^{k}+\delta \nu
\end{gathered}
$$

Due to the computational cost of integration over the (possibly high-dimensional) random space, an efficient implementation of the IRLS algorithm (section 4) and its Newton variant (section 5) is challenging. Hence, we next propose low-rank operator approximations that make these computations feasible. We also present estimates for the truncation error of these approximations and propose a diagonal preconditioner for the Newton step (42).

6. Low-rank operator approximations. Since we assume that the (possibly infinite-dimensional) uncertain parameter $m$ follows a normal distribution, (31), and equivalently (35), imply that, for given $\nu$, the corresponding control variable also follows a normal distribution. To be precise, if the distribution of $m$ is $\mu=\mathcal{N}\left(m_{0}, \mathcal{C}_{0}\right)$, then $u \sim \mathcal{N}\left(u_{\nu}, \mathcal{Q}_{\nu}\right)$ with

$$
u_{\nu}=S_{\nu} A^{-\star}\left(y_{d}-A^{-1}\left(f+B m_{0}\right)\right), \quad \mathcal{Q}_{\nu}=S_{\nu} A^{-\star} A^{-1} B \mathcal{C}_{0} B^{\star} A^{-1} A^{-\star} S_{\nu}^{\star},
$$

where $S_{\nu}$ is defined as in (36). Here, $S_{\nu}^{\star}$ is the adjoint operator of $S_{\nu}$ with respect to the $L^{2}$-inner product, but since $S_{\nu}$ is self-adjoint, $S_{\nu}=S_{\nu}^{\star}$.

In this section, we develop a method that exploits operator properties to enable the efficient implementation of the algorithms from sections 4 and 5. In particular, we use properties that are typical for instance for inverse elliptic PDE operators, to construct low-rank operator approximations. Moreover, we provide estimates for the resulting errors in terms of the truncated eigenvalues of the low-rank approximations.

6.1. Spectral decomposition of $\mathbf{A}^{-\star} \mathbf{A}^{-1}$ and truncation error analysis. We make the assumption that the symmetric and positive definite solution operator $A^{-\star} A^{-1}$ is a trace class operator, and thus its spectrum is rapidly decaying. This can be explored to enable fast computations based on low-rank approximations of the operator $S_{\nu}$, as discussed next. We denote by $D_{\nu}:=(\alpha+\beta \nu) I$, and assume we have a spectral decomposition of $A^{-\star} A^{-1}$ with decreasing eigenvalues $\lambda_{i}$ and corresponding eigenvectors $u_{i}, i \geq 1$. Thus,

$$
S_{\nu}=\left(A^{-\star} A^{-1}+D_{\nu}\right)^{-1}=\left(U \Lambda U^{\star}+D_{\nu}\right)^{-1},
$$

where $U^{\star} v=\left(\left\langle u_{i}, v\right\rangle_{L^{2}(\mathcal{D})}\right)_{i \geq 1} \in \ell_{2}$ for $v \in L^{2}(\mathcal{D})$ and $U \boldsymbol{y}=\sum_{i=1}^{\infty} y_{i} u_{i}$ for $\boldsymbol{y}=$ $\left(y_{i}\right)_{i \geq 1} \in \ell_{2}$, that is, $U$ and $U^{\star}$ are operators corresponding to a change of basis. 
Moreover, $\Lambda$ is a diagonal operator with entries $\lambda_{i}$. To approximate $\left(U \Lambda U^{\star}+D_{\nu}\right)^{-1}$, in the following, we will truncate the eigenvalue expansion of $A^{-\star} A^{-1}$. However, first we use the Sherman-Morrison-Woodbury formula and find

$$
S_{\nu}=\left(D_{\nu}+U \Lambda U^{\star}\right)^{-1}=D_{\nu}^{-1}-D_{\nu}^{-1}\left(U\left(\Lambda^{-1}+U^{\star} D_{\nu}^{-1} U\right)^{-1} U^{\star}\right) D_{\nu}^{-1} .
$$

To show how to control the approximation error resulting from eigenvalue truncation, we next derive an upper bound for the positive definite operator $S_{\nu}$. To compare positive definite operators $E$ and $F$, we say that $E \preccurlyeq F$ if $F-E$ is positive semidefinite. Then, because $\nu$ is positive, we have $U^{\star} D_{\nu}^{-1} U \preccurlyeq \alpha^{-1} I$, which implies that

$$
\Lambda^{-1}+\left(U^{\star} D_{\nu}^{-1} U\right) \preccurlyeq \Lambda^{-1}+\alpha^{-1} I=\operatorname{diag}\left(\frac{\lambda_{i}+\alpha}{\alpha \lambda_{i}}\right) .
$$

Consequently,

$$
\left(\Lambda^{-1}+\left(U^{\star} D_{\nu}^{-1} U\right)\right)^{-1} \succcurlyeq \operatorname{diag}\left(\frac{\alpha \lambda_{i}}{\lambda_{i}+\alpha}\right) .
$$

Along with (45), we conclude

$$
\left(D_{\nu}+U \Lambda U^{\star}\right)^{-1} \preccurlyeq D_{\nu}^{-1}-D_{\nu}^{-1}\left(U \operatorname{diag}\left(\frac{\alpha \lambda_{i}}{\lambda_{i}+\alpha}\right) U^{\star}\right) D_{\nu}^{-1} .
$$

Let us now consider an approximation of $A^{-\star} A^{-1}$ obtained by truncation of the eigenvalue expansion after the $r$ largest eigenvalues. The corresponding truncated analogues of $U$ and $\Lambda$ are denoted by $U_{r}$ and $\Lambda_{r}$, respectively. Then,

$$
\begin{aligned}
S_{\nu} & =\left(D_{\nu}+U_{r} \Lambda_{r} U_{r}^{\star}\right)^{-1}+R, \\
\text { where } \quad \frac{\operatorname{Tr}(R)}{\operatorname{Tr}\left(S_{\nu}\right)} & \leq \frac{\sum_{i=r+1}^{\infty} \frac{\lambda_{i}}{\lambda_{i}+\alpha}}{\sum_{i=1}^{\infty} \frac{\lambda_{i}}{\lambda_{i}+\alpha}} .
\end{aligned}
$$

Here, $\operatorname{Tr}(\cdot)$ denotes the operator trace. This shows that the contribution to the truncation error is small for eigenvalues that are small compared to $\alpha$. Note that the truncation error depends on $r$. This truncation error can be made arbitrary small by choosing $r$ large enough. This is of practical importance as it provides guidance on where to truncate the eigenvalue expansion. We obtain the following approximation $S_{\nu, r}$ of $S_{\nu}$ as in (45).

$$
S_{\nu, r}:=\left(D_{\nu}+U_{r} \Lambda_{r} U_{r}^{\star}\right)^{-1}=D_{\nu}^{-1}-D_{\nu}^{-1}\left(U_{r}\left(\Lambda_{r}^{-1}+U_{r}^{\star} D_{\nu}^{-1} U_{r}\right)^{-1} U_{r}^{\star}\right) D_{\nu}^{-1} .
$$

Note that the proposed algorithms will only require application of this operator to vectors, which can be done efficiently as will be discussed in subsection 7.3. To summarize, for given $\nu$, the corresponding optimal controls are normally distributed. Given a truncated eigenvalue expansion of $A^{-\star} A^{-1}$, this distribution can be approximated replacing $S_{\nu}$ by $S_{\nu, r}$ in (43). In the remainder of this section, we derive analogues for the gradient and Hessian of $\tilde{\mathcal{J}}$ building on the approximation $S_{\nu, r}$.

6.2. Gradient and IRLS using low-rank approximation. After characterizing the distribution of the optimal controls $u$, both the IRLS algorithm and its Newton variant require computation of $\|u\|_{\Omega}$, which involves integration over random space. To be precise, $\|u\|_{\Omega}=\|u\|_{\Omega}(\boldsymbol{x})$ is an integration over the Gaussian distribution $\mathcal{N}\left(u_{\nu}, \mathcal{Q}_{\nu}\right)$ with mean and covariance defined in (43), where $S_{\nu}$ is replaced by $S_{\nu, r}$. 
To approximate integration over the random space, we use the square root of $\mathcal{Q}$ given by $\mathcal{Q}^{1 / 2}=S_{\nu} A^{-\star} A^{-1} B \mathcal{C}_{0}^{1 / 2}$. Typical properties of $\mathcal{C}_{0}$ and $B$, as well as the trace class property of $A^{-\star} A^{-1}$ facilitate the approximation of $A^{-\star} A^{-1} B \mathcal{C}_{0}^{1 / 2}$ with rank- $\tilde{r}$ operators $E_{\tilde{r}} F_{\tilde{r}}^{\star}=\left[e_{1}, \ldots, e_{\tilde{r}}\right]\left[f_{1}, \ldots, f_{\tilde{r}}\right]^{\star}$ as follows:

$$
E_{\tilde{r}} F_{\tilde{r}}^{\star} \approx A^{-\star} A^{-1} B \mathcal{C}_{0}^{1 / 2} .
$$

Here, $e_{i} \in L^{2}(\mathcal{D})$ and $f_{i} \in \mathscr{H}$ which we can choose such that $f_{1}, \ldots, f_{\tilde{r}}$ are orthonormal. This results in the approximation

$$
\mathcal{Q}=\mathcal{Q}^{1 / 2}\left(\mathcal{Q}^{1 / 2}\right)^{\star} \approx S_{\nu, r} E_{\tilde{r}} E_{\tilde{r}}^{\star} S_{\nu, r}^{\star} .
$$

Thus, $\|u\|_{\Omega}^{2}$ can be approximated by $u_{\nu}(\boldsymbol{x})^{2}+\sum_{i=1}^{\tilde{r}}\left(S_{\nu, r} e_{i}\right)(\boldsymbol{x})^{2}$ for $\boldsymbol{x} \in \mathcal{D}$. Defining

$$
e_{0}:=A^{-\star}\left(y_{d}-A^{-1}\left(f+B m_{0}\right)\right)
$$

and recognizing that $u_{\nu}=S_{\nu, r} e_{0}$ allows the more compact notation

$$
\|u\|_{\Omega}^{2}(\boldsymbol{x}) \approx\|u\|_{\Omega, r}^{2}(\boldsymbol{x}):=\sum_{i=0}^{\tilde{r}}\left(S_{\nu, r} e_{i}\right)(\boldsymbol{x})^{2} .
$$

Using this in (38), we find the following approximation $\mathcal{G}_{r}(\nu)$ of the gradient of $\tilde{\mathcal{J}}$ :

$$
\mathcal{G}(\nu) \approx \mathcal{G}_{r}(\nu):=\sum_{i=0}^{\tilde{r}}\left(S_{\nu, r} e_{i}\right)^{2}+\varepsilon^{2}-\frac{1}{\nu^{2}}
$$

and a very similar expression to update the reweighting function in the IRLS method.

6.3. Hessian using low-rank approximation. We now derive expressions for the application of the Hessian corresponding to the gradient $\mathcal{G}_{r}(\nu)$ to vectors. We do this by taking derivatives of $\mathcal{G}_{r}(\nu)$ with respect to $\nu$ in a direction $\delta \nu$. This results in the following Hessian $\mathcal{H}_{r}$ based on the low-rank approximation,

$$
\mathcal{H}(\nu) \delta \nu \approx \mathcal{H}_{r}(\nu) \delta \nu:=-2 \beta \sum_{i=0}^{\tilde{r}}\left(S_{\nu, r} e_{i}\right) \odot S_{\nu, r}\left(\left(S_{\nu} e_{i}\right) \odot \delta \nu\right)+\frac{2}{\nu^{3}} \odot \delta \nu,
$$

where we have used the identity $\left(S_{\nu, r} w\right)_{, \nu} \tilde{\nu}=-\beta S_{\nu, r}\left(\left(S_{\nu, r} w\right) \odot \tilde{\nu}\right)$. To summarize, a Newton step based on the low-rank approximation of $A^{-\star} A^{-1}$ is as follows:

$$
\begin{aligned}
\mathcal{H}_{r}\left(\nu^{k}\right) \delta \nu & =-\mathcal{G}_{r}\left(\nu^{k}\right), \\
\nu^{k+1} & =\nu^{k}+\delta \nu .
\end{aligned}
$$

Despite the low-rank approximation, $\mathcal{H}_{r}$ is usually not explicitly available. Hence, this Newton system must be solved using an iterative method that, such as the conjugate gradient method, only requires the application of $\mathcal{H}_{r}\left(\nu^{k}\right)$ to vectors.

6.4. Preconditioning of Newton system. The convergence of the conjugate gradient (CG) method in each Newton step depends crucially on the availability of an effective preconditioner. This is particularly true if the Hessian operator is very illconditioned, as is the case due to the $1 / \nu^{3}$ term in $\mathcal{H}_{r}$, which can vary over many orders of magnitude if $\varepsilon$ is small. Using the low-rank approximations established above, 
we propose a diagonal preconditioner that is effective in practice, as we illustrate numerically in section 8 . The diagonal of the Hessian $\mathcal{H}_{r}(\nu)$ is given by

$$
\mathcal{P}_{\operatorname{diag}}(\nu)=-2 \beta \operatorname{diag}\left(S_{\nu, r}\right) \sum_{i=1}^{\tilde{r}}\left(\left(S_{\nu, r} e_{i}\right) \odot\left(S_{\nu, r} e_{i}\right)\right)+\frac{2}{(\nu)^{3}} .
$$

Here, $\operatorname{diag}\left(S_{\nu, r}\right)$ is the diagonal of $S_{\nu, r}$, which can be computed from (47) using that

$$
\operatorname{diag}\left(U_{r}\left(\Lambda_{r}^{-1}+U_{r}^{\star} D_{\nu}^{-1} U_{r}\right)^{-1} U_{r}^{\star}\right)=\sum_{i=1}^{r} u_{i} \odot w_{i},
$$

where $w_{i}=\left(\Lambda_{r}^{-1}+U_{r}^{\star} D_{\nu}^{-1} U_{r}\right)^{-1} u_{i}$. Note that the terms $S_{\nu, r} e_{i}$ in (54) are already available from the gradient computation. Moreover, this diagonal preconditioner depends on $\nu$, which means it must be recomputed for each Newton step.

7. Offline-online algorithms for shared sparsity control problem. The algorithms presented in this section are the result of combining the norm reweighting algorithms from sections 4 and 5 with the low-rank approximations from section 6. For large-scale and thus computationally challenging problems, our method can be split into an offline phase, itself consisting of a setup and a compute step, and an online phase. The offline phase includes a setup step, in which we construct a low-rank approximation for the PDE-solution operator $A^{-\star} A^{-1}$ and the operator $A^{-\star} A^{-1} B \mathcal{C}_{0}^{1 / 2}$. This is followed by the offline compute step, in which we solve the optimization problem (14) or (21). In this step, one can adjust the weight $\beta>0$ for the sparsity-enhancing term in the objective to obtain the desired sparsity structure which, in applications, depends on the availability of control devices. In the online phase, the goal is to compute the optimal control for a specific (and known) realization of the uncertain parameter $m(\omega)$. Here, one can use the low-rank approximation for the fast computation of the optimal control for a specific event $\omega$. In this step, the sparsity structure and operator approximations determined in the offline phase are used.

7.1. Offline phase. In the offline phase, we first compute a rank- $r$ approximation of the positive self-adjoint operator $A^{-\star} A^{-1}$, i.e.,

$$
A^{-\star} A^{-1} \approx U_{r} \Lambda_{r} U_{r}^{\star} .
$$

As shown in the previous section, the error in the optimal control solution due to truncation is small when the truncated eigenvalues are small compared to $\alpha$ - see (46). The low-rank approximation can be found using either the Lanczos method [34], or a randomized algorithm [20]. These methods only require the application of the linear operator $A^{-*} A^{-1}$ to vectors, i.e., each application amounts to a solve with the forward and the adjoint PDE operators $A$ and $A^{\star}$. Next, we compute $e_{0}$ according to (50) and compute a rank- $\tilde{r}$ approximation of $A^{-\star} A^{-1} B \mathcal{C}_{0}^{1 / 2}$ as follows:

$$
E_{\tilde{r}} F_{\tilde{r}}^{\star} \approx A^{-\star} A^{-1} B \mathcal{C}_{0}^{1 / 2},
$$

where one can use the low-rank approximation (56). In most practical applications, $B \mathcal{C}_{0}^{1 / 2}$ has a fast decaying spectrum since $\mathcal{C}_{0}$ is a trace class operator and thus, typically, $\tilde{r}<r$. After these preparations, we are ready to either employ the IRLS algorithm (Algorithm 1) or its Newton variant (Algorithm 2). Note that in Algorithm 1, the expression in the termination criterion (Line 6) is the norm of the reduced gradient (52) due to the relation (40). 

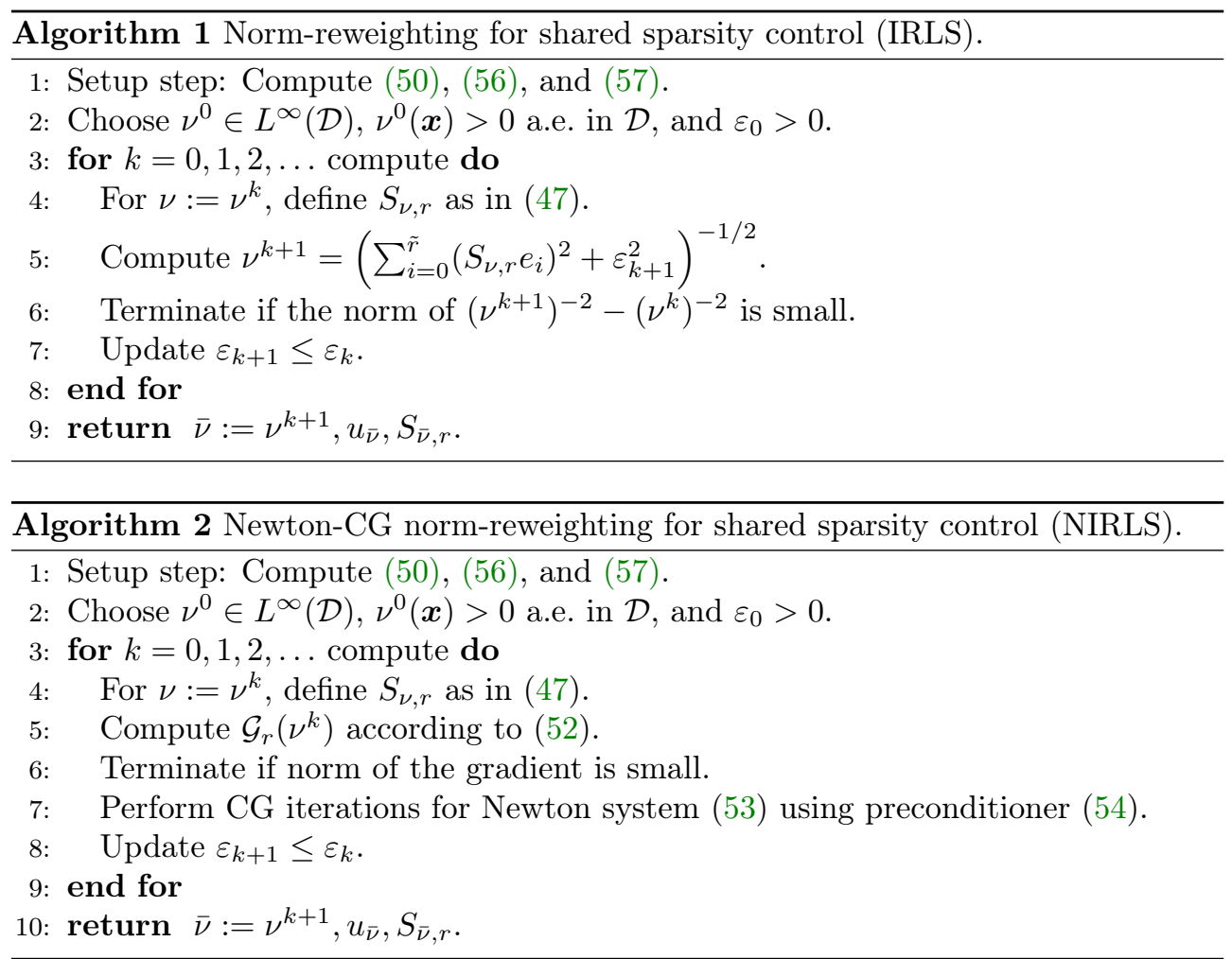

7.2. Online phase. During the online phase, we compute the optimal control for a specific realization of the uncertain parameter $m(\omega)$. This step uses the weight function $\bar{\nu}$ found in the offline phase, and also uses the truncated spectral expansion (46). To be precise, for a sample draw $m(\hat{\omega})=m_{0}+\hat{m}(\omega)$ from $\mathcal{N}\left(m_{0}, \mathcal{C}_{0}\right)$, the corresponding optimal control $\hat{u} \in V$ is computed as

$$
\hat{u}=S_{\bar{\nu}, r}\left(e_{0}-U_{r} \Lambda_{r} U_{r}^{\star} B \hat{m}\right),
$$

where $\bar{\nu}$ and $S_{\bar{\nu}, r}$ are as returned by Algorithm 1 or Algorithm 2. Note that the optimal control $\hat{u}$ has the sparsity structure determined in the offline phase.

7.3. Computational cost. Here, we summarize and compare the dominant computational cost of the proposed algorithms. We denote by $N$ the discretization dimension of the state and control variable, and discuss the complexity of the offline phase (setup and optimization steps) and the online phase (computation of optimal control). In the arguments below, we assume that $\tilde{r} \leq r \ll N$.

Offline phase: Setup. In the offline phase, we first compute the truncated spectral approximation (56). This requires solves with $A$ and the adjoint $A^{\star}$. The number of required solves depends on the spectrum of the operator $A^{-\star} A^{-1}$, on the value of $\alpha$ and on the truncation error one is willing to commit. The reason why we report the complexity in terms of PDE solves is that the cost in terms of operations depends on whether $A$ is available as assembled matrix, which solvers are applicable to solve systems with $A$ and $A^{*}$, and which solvers are available to a user. To compute a rank- $r$ approximation usually requires $r+d$ products with $A^{-\star} A^{-1}$, where $d$ is small (e.g., 10) to obtain a accurate rank- $r$ approximation. If the low-rank approximation is computed 
using a randomized singular value decomposition, then $d$ is the oversampling factor [20]. In the Lanczos method, adding $d$ iterations enriches the Krylov space and thus leads to improved accuracy of the dominant directions. It remains to estimate the computational work for computing a singular value decomposition of $A^{-\star} A^{-1} B \mathcal{C}_{0}^{1 / 2}$, required to find $E_{\tilde{r}}=\left[e_{1}, \ldots, e_{\tilde{r}}\right]$, during the offline phase. This step can build on the truncated spectral decomposition of $A^{-\star} A^{-1}$ and thus does not require additional PDE solves. Hence, the complexity of the offline setup phase is $2(r+d)$ PDE solves.

Offline phase: Optimization. After the above setup step, the remaining steps do not require further PDE solves and we simply estimate the complexity of the proposed algorithms in terms of elementary linear algebra operations. Let us first consider the computations required in each iteration of IRLS (Algorithm 1). Note that each step is equivalent to computing (38), the gradient $\mathcal{G}_{r}$ of the reduced objective $\tilde{J}$. Thus, the computational complexity of one IRLS step coincides with computing the right hand side for the Newton step (42).

Computing $\mathcal{G}_{r}$ requires application of the operator $S_{\nu, r}$, defined in (47), to vectors. First, this necessitates the inverse of the $r \times r$ matrix $\left(\Lambda_{r}^{-1}+U_{r}^{*} D_{\nu}^{-1} U_{r}\right)$. This step is dominated by the computation of $U_{r}^{*} D_{\nu}^{-1} U_{r}$, which amounts to $r^{2} N$ operations. Since we assume that $N \gg r$, this dominates computation of an $r \times r$ matrix inverse. Each application of $S_{\nu, r}$ to a vector requires $2 r N$ operations, amounting overall to a complexity of $2 r N(\tilde{r}+1)$ operations to compute $\|u\|_{\Omega}$. Thus, for the IRLS algorithm, the computational complexity per iteration is $r N(r+2 \tilde{r})$.

Additionally to the computation of the gradient $\mathcal{G}_{r}$, each iteration of the NIRLS method (Algorithm 2) requires the application of the Hessian to one vector in each CG step, amounting to $2 \tilde{r} r N$ operations. It also requires to setup the preconditioner matrix, which requires $r^{2} N$ operations as can be seen from (55). Hence, we find that the computational complexity for one inexact Newton-CG step is $2 r N\left(r+\tilde{r}+\tilde{r} n_{\mathrm{cg}}\right)$, where $n_{\text {cg }}$ denotes the number of CG iterations.

Note that it depends on $r, \tilde{r}$ how much larger the complexity of a NIRLS iteration is than an IRLS iteration. If $\tilde{r}$ is significantly smaller than $r$, as in the example problems in section 8, one CG step only amounts to a fraction of the complexity of one IRLS step. Finally, note that all steps in the offline optimization algorithms have optimal complexity, i.e., they depend linearly on the discretization dimension $N$.

Online phase. In the online phase, the only necessary computation is (58). For any fixed sample draw $\hat{m}(\omega)$, this is a deterministic expression. This online step requires multiplication of a vector with $U_{r}$ and its transpose, and it requires one application of $S_{\nu, r}$ to a vector. This amounts to overall about $4 r N$ operations, and can thus be done fast and potentially in real-time, depending on the application.

8. Numerical experiments. We end this paper with a numerical study for the stochastic control problem with shared sparsity. Our aims are to study the qualitative effect of the shared sparsity term on the optimal controls (subsection 8.1), and to investigate the performance and accuracy of the proposed algorithms (subsection 8.2).

For this purpose, we use three model problems, which all use the physical domain $\mathcal{D}=(0,1)^{2} \subset \mathbb{R}^{2}$. The boundary is split in $\partial D_{2}=\{0\} \times[0,1]$ and $\partial D_{1}=\partial D \backslash \partial D_{2}$. A simple finite difference approximation (i.e., the five-point stencil) on a mesh of $n \times n$ points is used to discretize the Laplacian that is part of the differential operator $A$. While both algorithms we propose allow for a decreasing sequence of positive values $\varepsilon_{1} \geq \varepsilon_{2} \geq \ldots$, we fix $\varepsilon$ to a small value in our tests, and study the influence of that value on the performance of the methods.

Problem 9. This first problem is of the form of Example 1 with $k=0$ and 

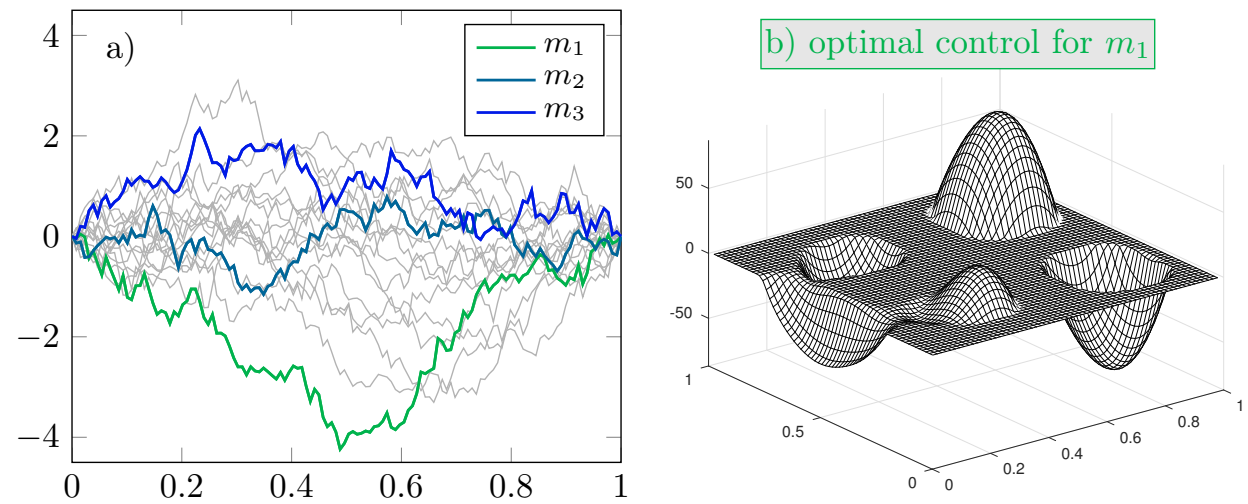

c) optimal control for $m_{2}$

d) optimal control for $m_{3}$
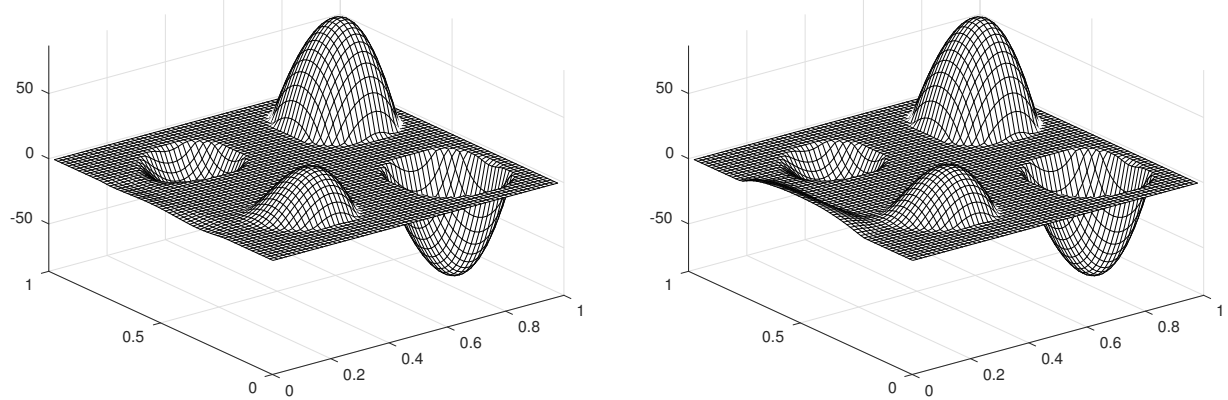

FIG. 1. Results for Problem 9: Random draws for boundary Neumann data (a). The highlighted samples are used to compute the optimal controls shown in (b), (c) and (d). Note that the controls are different but share the same sparsity structure.

$a(\boldsymbol{x}) \equiv 1$. Except for the uncertain Neumann boundary data, it coincides with Example 1 from [35]. In particular, the PDE-operator is $A=-\Delta$ with zero Dirichlet boundary conditions on $\partial D_{1}$ and Neumann boundary conditions on $\partial D_{2}$. Further, $y_{d}=\sin (2 \pi x) \sin (2 \pi y) \exp (2 x) / 6, f \equiv 0, \alpha=10^{-5}$ and $\beta=10^{-3}$. The uncertain parameter field enters as Neumann data on $\partial D_{2}$. This data follows an infinitedimensional Gaussian distribution with mean $m_{0} \equiv 0$. The covariance operator is given as the inverse elliptic PDE operator $\mathcal{C}_{0}=\gamma\left(-\partial_{\boldsymbol{x} \boldsymbol{x}}\right)^{-1}$, with homogeneous Dirichlet boundary conditions at the boundary of $\partial D_{2}$, i.e., at the two points $(0,0)$ and $(1,0)$, and with $\gamma=4$. It can easily be verified that $\mathcal{C}_{0}$ is a symmetric and positive definite trace-class operator, and thus defines a valid covariance operator [15]. Random draws from this distribution are shown in Figure 1a, and optimal controls in the remaining figures in Figure 1.

Problem 10. This problem has the form of Example 2. The data are as in Problem 9 , but the uncertainty enters on the right hand side of the equation rather than as Neumann boundary data, and $\alpha=5 \times 10^{-5}$. The uncertain parameter $m$ is distributed as an infinite-dimensional Gaussian random field over the two-dimensional physical domain $\mathcal{D}$. Its mean is $m_{0} \equiv 0$ and the covariance operator is the squared inverse elliptic PDE operator $\mathcal{C}_{0}=\gamma(-\Delta)^{-2}$, where $\gamma=20^{2}$ and the Laplace operator $\Delta$ in $\mathcal{C}_{0}$ satisfies homogeneous Dirichlet conditions on $\{1\} \times[0,1]$ and homogeneous 

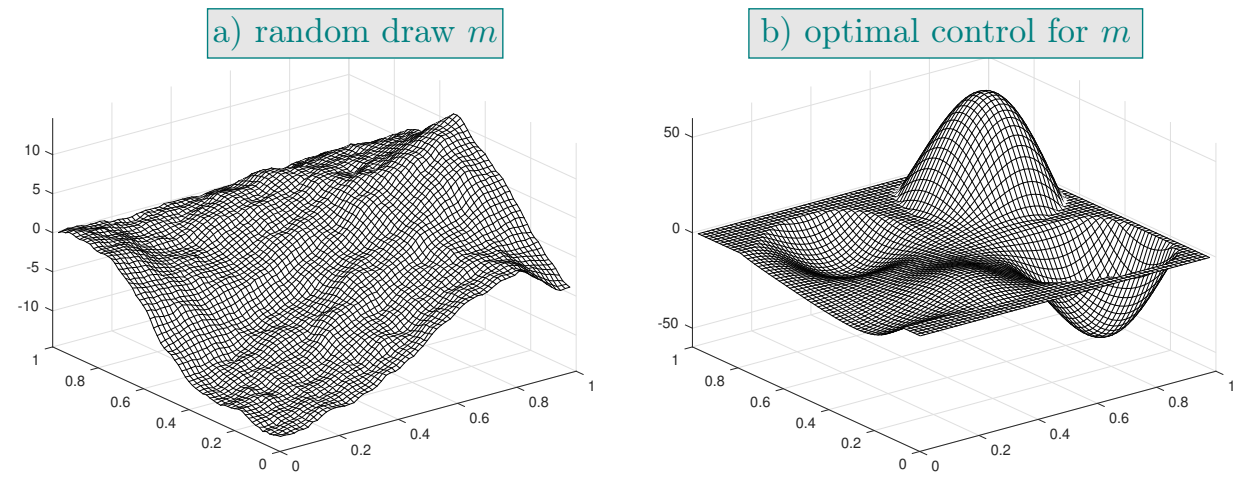

FIG. 2. Results for Problem 10: Shown in (a) is a random draw from the Gaussian random field defined over $\mathcal{D}$. Note that all draws of the random field satisfy a homogeneous Dirichlet condition on part of the boundary. The figure (b) shows the corresponding optimal control.

Neumann conditions for the remaining boundaries. $\mathcal{C}_{0}$ is a valid covariance operator on $L^{2}(\mathcal{D})$ as it is symmetric, positive and trace-class [15]. A random draw from this distribution and the corresponding optimal control are shown in Figure 2.

Problem 11. This problem has the form of Example 3. In particular, $A=-\Delta-$ $\kappa^{2} I$ with $\kappa=12$ is the indefinite Helmholtz operator. Moreover, $f \equiv 0$ and $y_{d} \equiv 0$, i.e., our aim is to dampen the uncertain Neumann boundary forcing, whose distribution is as in Problem 9. Optimal controls for $\alpha=5 \times 10^{-5}$ and $\beta=5 \times 10^{-4}$ are shown in Figure 3. This problem is a substantially simplified version of the earthquake engineering/vibration damping problem given as example in the introduction, where one aims to find controller locations that are best at actively dampening waves originating from uncertain boundary forcing. Clearly, this example only uses a single frequency and a simple model for wave propagation.

8.1. Qualitative solution properties. Let us first discuss the results of Problem 9 shown in Figure 1. As in the version of this problem not involving uncertain parameters [35], the distributed controls vanish on parts of the domain. We also find that increasing $\beta$ increases sparsity (not shown here). The deterministic optimal control, i.e., the solution to (13), looks similar to the stochastic optimal controls from Figure 1 but vanishes near the Neumann boundary. As expected for the stochastic control problem, the optimal controls corresponding to different realizations of the uncertain Neumann data differ, but they share the same sparsity structure. Note that the differences between the optimal controls occur primarily close to the boundary $\partial \mathcal{D}_{2}$, which is where the uncertain Neumann data enter in the problem. This local effect of different Neumann data is due to the locality properties of the Laplace operator. A different behavior is found for Problem 11. Here, as can be seen from Figure 3, the optimal controls differ substantially even far away from $\partial \mathcal{D}_{2}$, which is a consequence of the non-local behavior of Helmholtz equation solutions. In fact, in this problem, the only cause for the controls to be nonzero is the Neumann boundary data, which impacts the optimal controls in a non-local manner. We also point out that the solution of the deterministic version (13) of Problem 11 is zero.

8.2. Performance of algorithms. Next, we focus on the accuracy of the lowrank approximations we employ and the quantitative behavior of our solution algo- 

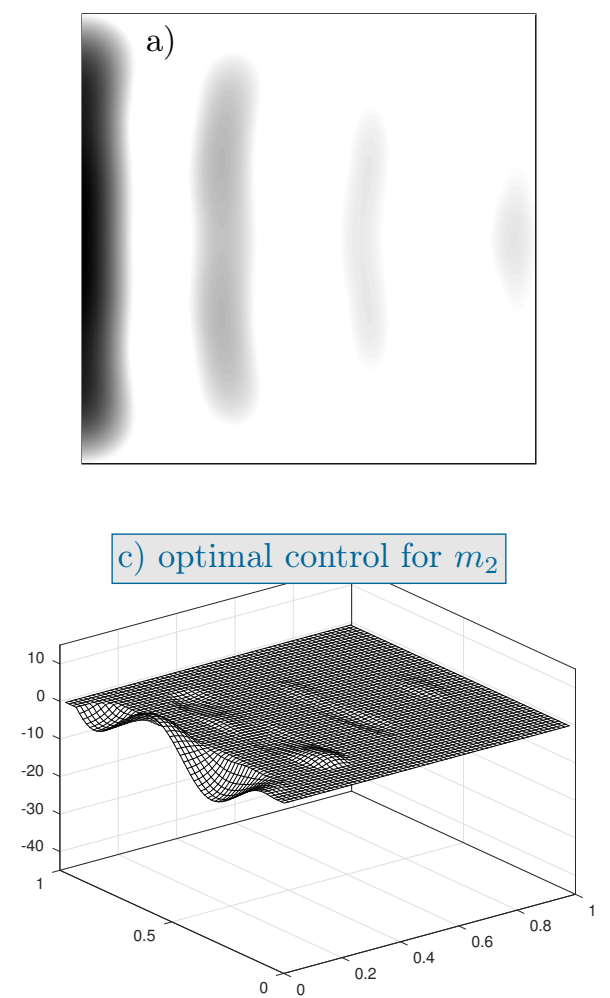
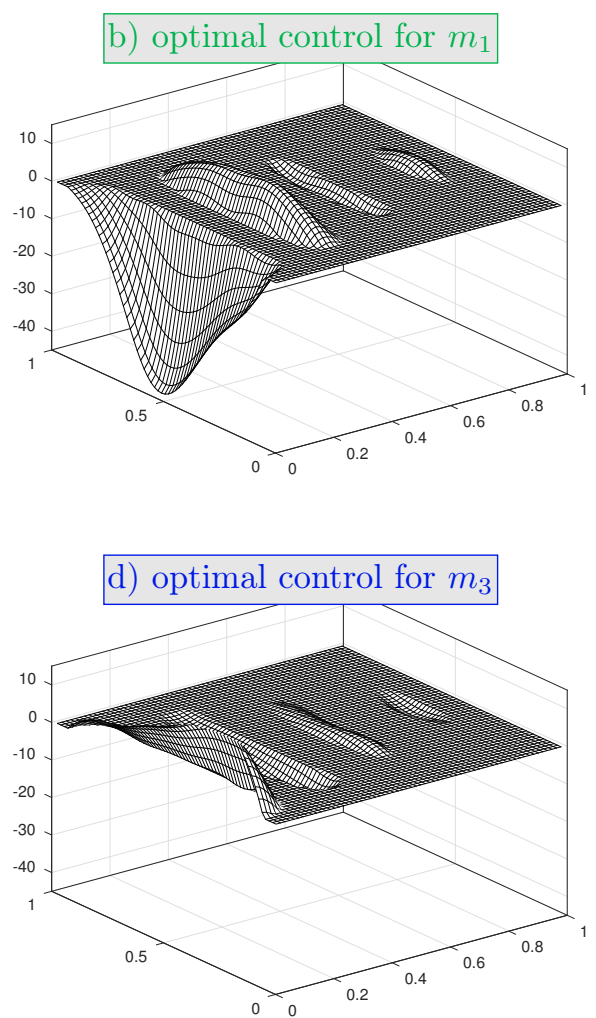

FIG. 3. Results for Problem 11: Shown in (a) is the pointwise standard deviation $\nu^{-1}$ of the optimal controls. All stochastic controls have their support in the gray regions, and vanish in the white regions. The figures (b), (c) and (d) show the optimal controls corresponding to the same samples of the uncertain Neumann boundary condition highlighted in Figure 1 (a). Note that the optimal controls are different but have the same sparsity structure.

rithms. While first we compare the low-rank approximations for both, Problems 9 and 11, the remainder of the results shown in this section are for Problem 11. We have verified that the behavior of the algorithms for Problem 9 is similar.

Truncation and low-rank approximation. Let us first discuss the low-rank approximation of $A^{-\star} A^{-1}$ and the choice of the truncation. In Figure 4, the spectra of $A^{-\star} A^{-1}$ for Problems 9 and 11 are shown for different mesh resolutions. First, it can be seen that the eigenvalues converge as the mesh is refined, which is a consequence of the smoothness of the eigenvectors of the inverse Laplace and Helmholtz operators. Second, these plots help determine a reasonable truncation for the low-rank approximation. For that purpose, we recall that from the error term in (46) it follows that the low-rank error depends on how small the truncated eigenvalues are compared to the value of $\alpha$ (also shown in Figure 4. For our numerical results, we use low-rank approximations with $r=180$ for Problem 9 and Problem 10, $r=150$ for Problem 11. For each example, we show the exact relative truncation error at the solution and the upper bound (46b), in which we truncate the infinite sums after another 500 eigenvalues. For Problem 9, the relative truncation error rate is $3.3 \times 10^{-6}$ while the estimation is $3.5 \times 10^{-2}$. In Problem 11, we have a relative truncation error of $1.5 \times 10^{-4}$ and the theoretical bound is $3.0 \times 10^{-2}$. For the problems with the uncertain 

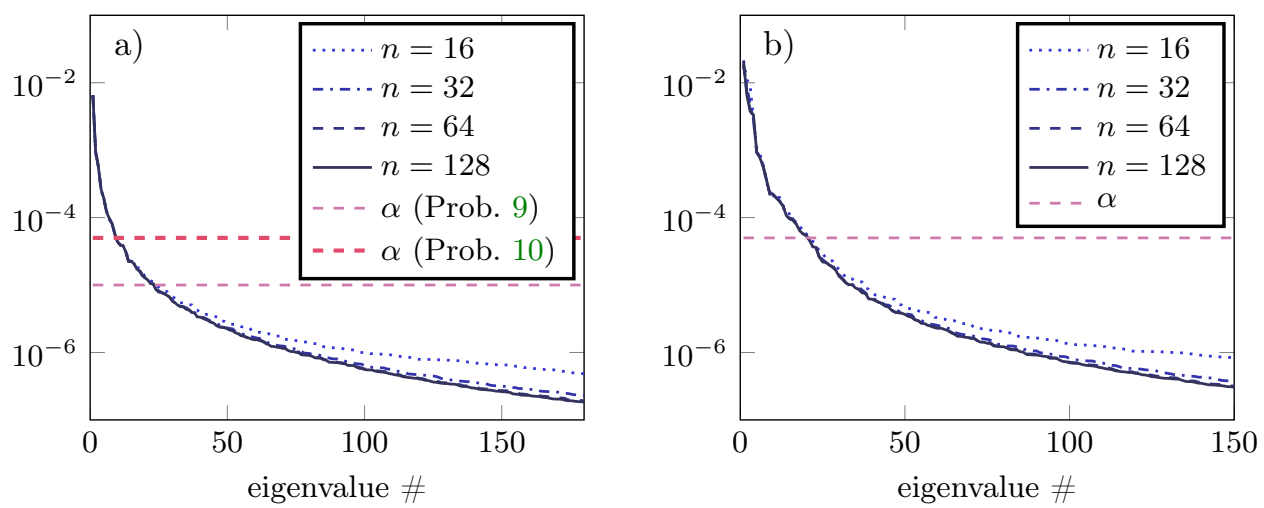

Fig. 4. Spectra of $A^{-\star} A^{-1}$ for discretizations with $n \times n$ points for Problem 9, Problem 10 (a) and Problem 11 (b). As reference, we also show the value of $\alpha$, which allows estimation of the truncation error as discussed in subsection 6.1.

boundary data, $\tilde{r}=16$ results in approximation of (48) up to machine precision. For Problem 10, $\tilde{r}=64$ is used since the two-dimensional Gaussian random field requires more approximation vectors. This $\tilde{r}$ captures $>99.9 \%$ of (48). We have numerically verified that the convergence behavior does not change substantially if higher-rank approximations are used. Moreover, the optimal controls are visually identical when more basis functions are used, showing that the error due to truncation is small.

IRLS and over-relaxed IRLS. In Figure 5a, we show the performance of Algorithm 1. We attempt to speed up the algorithm by means of over-relaxation, using the reweighting function

$$
\nu^{k+1}:=(1-\theta) \nu^{k}+\theta \bar{\nu}^{k+1},
$$

where $\theta \geq 1$ and $\bar{\nu}^{k+1}$ is the weight function computed from the (original) IRLS algorithm. For $\theta=1$, we recover the original method. Empirical experiments have led us to choose $\theta=1.5$, which leads to moderately faster convergence, as can be seen in Figure 5a. The IRLS algorithms converge rather slowly but monotonously, as predicted by the theory and also observed in other contexts [16,24]. We find the convergence behavior of the IRLS algorithm to be largely independent of $\varepsilon$ and of the discretization mesh size $N$.

NIRLS algorithm. Next, we study the performance of the preconditioned NewtonCG algorithm (Algorithm 2). Since the Newton method is not guaranteed to converge monotonously and the IRLS algorithm converges rapidly in early iterations, we first perform 15 over-relaxed IRLS steps, and then switch to NIRLS. Figure 5b-d shows performance results of the method for different numbers of preconditioned CG iterations per Newton step, various values of $\varepsilon$ and different discretizations. In Figure 5b,c, we show the norm of the gradient versus the computational cost as discussed in subsection 7.3. We choose one step of the IRLS algorithm (i.e., one computation of the gradient $\mathcal{G}_{r}$ ) as the unit of cost. The computational complexity of NIRLS iterations is converted to this cost unit to allow for a fair comparison between the different methods. For instance, following the complexity estimates from subsection 7.3 and using the specific choices $r=150$ and $\tilde{r}=16$ used in this problem, the computational complexity of a step of NIRLS with 3 CG iterations is $2.35 \times$ the cost of one IRLS iteration. This ratio increases to 3.23 if 8 CG iterations are used in each NIRLS step. As can be seen in Figure 5b, the NIRLS method converges significantly faster than the 
IRLS method. Moreover, a small number of CG steps per Newton iteration results in the fastest convergence in terms of computational cost. Thus, we use 3 CG steps per Newton iteration for the remaining tests. Figure $5 \mathrm{c}$ shows that we observe fast local convergence for every value of $\varepsilon$ and that we observe a mild dependence of the convergence on the value of $\varepsilon$. Finally, Figure $5 \mathrm{~d}$ compares the convergence for different mesh discretizations and we observe mesh-independent convergence behavior, which illustrates the efficiency of the diagonal preconditioner.
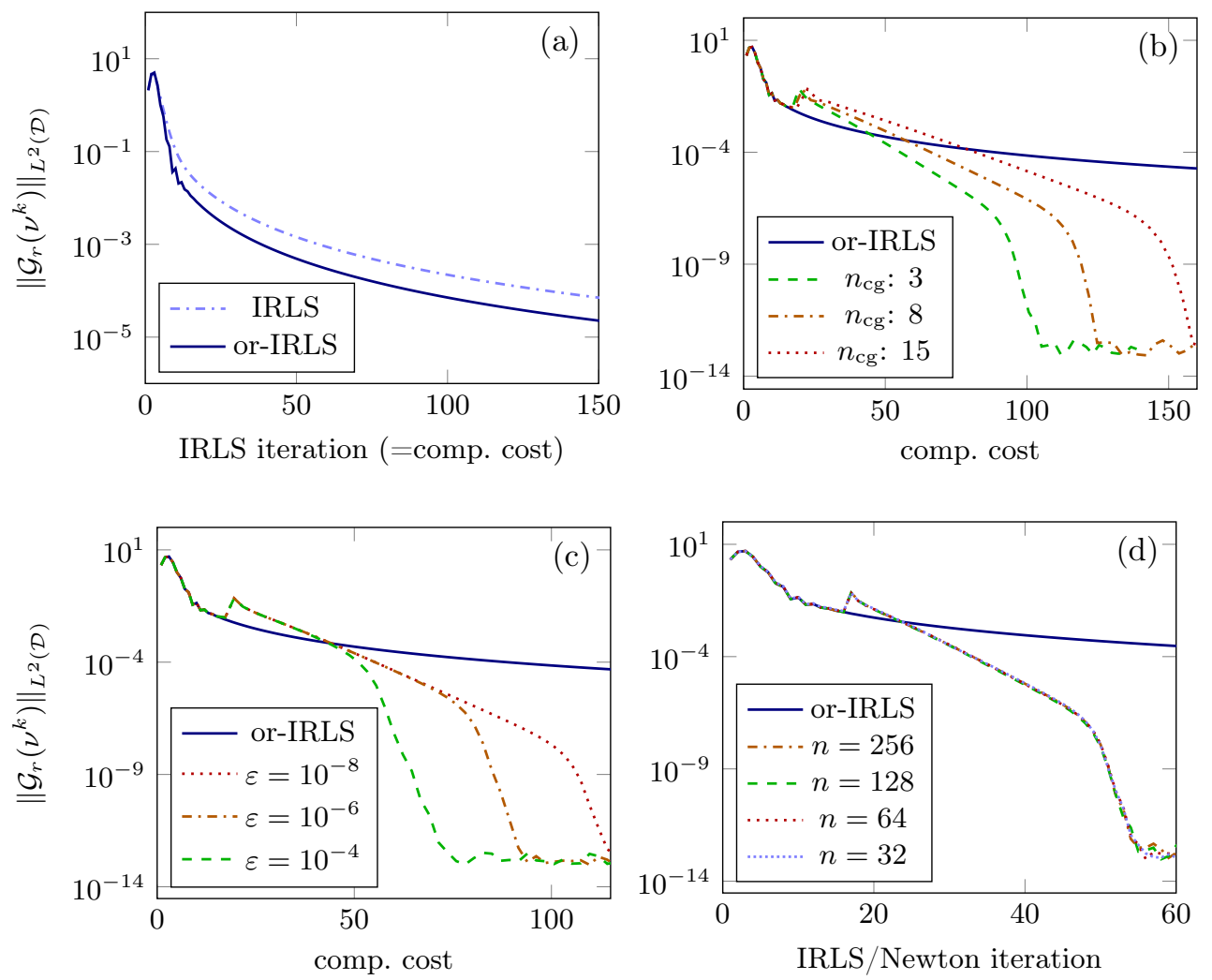

FIG. 5. Convergence behavior of algorithms for Problem 11. Shown in (a) is the reduction of the norm of the gradient for IRLS and over-relaxed IRLS (or-IRLS) with $\theta=1.5$ (see (59)). Shown in (b) is a comparison of the performance of or-IRLS and the preconditioned Newton-CG method NIRLS for different numbers of CG iterations per Newton step, where we fix $n=128, \varepsilon=10^{-7}$. The figure in (c) compares the convergence of the preconditioned Newton-CG method with different $\varepsilon$ for $n=128$ and $3 C G$ iterations per Newton step. Shown in (d) is a comparison of the convergence of or-IRLS and the NIRLS method for different mesh sizes $n, \varepsilon=10^{-7}$ and $3 C G$ iterations per Newton step. As discussed in subsection 7.3, in (a)-(c), we use the computational work required for one IRLS iteration as unit for the x-axis to compare the computational complexity of the IRLS algorithms and its Newton variants. In (d), we use the iteration number as unit for the $x$-axis since we study how the number of iterations changes for different discretizations.

9. Discussion and remarks. First, let us discuss the role of $\alpha>0$. This parameter plays a significant role in our problem formulation, the proposed solution algorithms and their analysis. Positivity of $\alpha$ is required for the deterministic problem to be formulated in an $L^{2}$-Hilbert space framework rather than over a space of measures [8,33]. Additionally, $\alpha>0$ plays a crucial role for the truncation of the spectral expansion of $A^{-\star} A^{-1}$ since we show that the truncation error is small when 
the truncated eigenvalues are small compared to $\alpha$. Both aspects are related to the regularizing effect positive values of $\alpha$ have on the controls.

Second, it would be desirable to include control bounds in the proposed algorithms. However, it is not obvious how to achieve this without resorting to more general algorithms that also apply to nonlinear problems and use random space approximations, such as stochastic Galerkin/collocation or Monte Carlo methods. The main difference between bound constraints and the shared sparsity term is that bound constraints apply to the controls individually for each random event, while the sparsity term involves integration over the probability space.

Third, the proposed approach can be generalized to uncertain parameters that do not follow a Gaussian distribution. As long as for the resulting distribution of the controls, $\|u\|_{\Omega}(\boldsymbol{x})$ can be computed efficiently, the reweighting algorithms can be applied to compute jointly sparse controls.

We believe that several questions raised in this paper deserve further research. For instance, while challenging, extension to nonlinear problems are worthwhile pursuing, as well as the question whether the shared sparsity requirement can help to reduce the effective dimension of non-linear problems. Other interesting questions include a study of the spatial discretization of the problem, extensions to parabolic governing equations possibly combined with directional sparsity, problem formulation and algorithms for $\alpha=0$, and the question whether regularization with $\varepsilon>0$ can be avoided. A question of potentially general interest is if the Newton variant of the IRLS algorithm can accelerate the solution of other non-smooth optimization problems for which currently reweighting algorithms are used.

Acknowledgments. The authors would like to thank two anonymous referees for their thoughtful comments and suggestions. GS would like to additionally thank Noemi Petra, Alen Alexanderian and Kazufumi Ito for valuable discussions.

\section{REFERENCES}

[1] A. Alexanderian, N. Petra, G. Stadler, and O. Ghattas, Mean-variance risk-averse optimal control of systems governed by PDEs with random parameter fields using quadratic approximations, SIAM/ASA Journal on Uncertainty Quantification, 5 (2017), pp. 11661192, https://doi.org/10.1137/16M106306X. arXiv preprint arXiv:1602.07592.

[2] A. A. Ali, E. Ullmann, And M. Hinze, Multilevel Monte Carlo analysis for optimal control of elliptic PDEs with random coefficients, SIAM/ASA J. Uncertainty Quantification, 5 (2017), pp. 466-492, https://doi.org/10.1137/16M109870X.

[3] P. Benner, A. Onwunta, And M. Stoll, Block-diagonal preconditioning for optimal control problems constrained by pdes with uncertain inputs, SIAM Journal on Matrix Analysis and Applications, 37 (2016), pp. 491-518, https://doi.org/10.1137/15M1018502.

[4] A. Borzì And G. von Winckel, A POD framework to determine robust controls in PDE optimization, Computing and Visualization in Science, 14 (2011), pp. 91-103.

[5] P. Brunner, C. Clason, M. Freiberger, and H. Scharfetter, A deterministic approach to the adapted optode placement for illumination of highly scattering tissue, Biomedical Optics Express, 3 (2012), pp. 1732-1743, https://doi.org/10.1364/BOE.3.001732.

[6] E. J. Candes, M. B. Wakin, And S. P. Boyd, Enhancing sparsity by reweighted $l^{1}$ minimization, Journal of Fourier Analysis and Applications, 14 (2008), pp. 877-905, https://doi.org/10.1007/s00041-008-9045-x.

[7] M. Caponigro, M. Fornasier, B. Piccoli, and E. Trélat, Sparse stabilization and control of alignment models, Mathematical Models and Methods in Applied Sciences, 25 (2015), pp. 521-564.

[8] E. CASAS, A review on sparse solutions in optimal control of partial differential equations, SeMA Journal, 74 (2017), pp. 319-344, https://doi.org/10.1007/s40324-017-0121-5.

[9] E. Casas, C. Clason, and K. Kunisch, Approximation of elliptic control problems in measure spaces with sparse solutions, SIAM Journal on Control and Optimization, 50 (2012), 
pp. $1735-1752$

[10] E. Casas, C. RYll, and F. TröLtzsch, Sparse optimal control of the Schlögl and FitzhughNagumo systems, Computational Methods in Applied Mathematics, 13 (2013), pp. 415442.

[11] P. Chen and A. Quarteroni, Weighted reduced basis method for stochastic optimal control problems with elliptic PDE constraint, SIAM/ASA Journal on Uncertainty Quantification, 2 (2014), pp. 364-396.

[12] G. Ciaramella And A. Borzi, Quantum optimal control problems with a sparsity cost functional, Numerical Functional Analysis and Optimization, 37 (2016), pp. 938-965, https://doi.org/10.1080/01630563.2016.1184166.

[13] C. Clason and K. Kunisch, A measure space approach to optimal source placement, Computational Optimization and Applications, 53 (2012), pp. 155-171.

[14] G. Da Prato and J. Zabczyk, Second-order partial differential equations in Hilbert spaces, Cambridge University Press, 2002.

[15] G. Da Prato and J. Zabczyk, Stochastic equations in infinite dimensions, Cambridge University Press, 2014.

[16] I. Daubechies, R. DeVore, M. Fornasier, and C. S. Güntürk, Iteratively reweighted least squares minimization for sparse recovery, Communications on Pure and Applied Mathematics, 63 (2010), pp. 1-38.

[17] I. Ekeland and R. Temam, Convex Analysis and Variational Problems, Classics in Applied Mathematics, Vol. 28, SIAM, Philadelphia, 1999.

[18] M. Fornasier, S. Peter, H. Rauhut, and S. Worm, Conjugate gradient acceleration of iteratively re-weighted least squares methods, Computational Optimization and Applications, 65 (2016), pp. 205-259, https://doi.org/10.1007/s10589-016-9839-8.

[19] S. Garreis And M. Ulbrich, Constrained optimization with low-rank tensors and applications to parametric problems with pdes, SIAM Journal on Scientific Computing, 39 (2017), pp. A25-A54, https://doi.org/10.1137/16M1057607.

[20] N. Halko, P. G. Martinsson, And J. A. Tropp, Finding structure with randomness: Probabilistic algorithms for constructing approximate matrix decompositions, SIAM Review, 53 (2011), pp. 217-288.

[21] R. Herzog, G. Stadler, and G. Wachsmuth, Directional sparsity in optimal control of partial differential equations, SIAM Journal on Control and Optimization, 50 (2012), pp. 943963, https://doi.org/10.1137/100815037, http://link.aip.org/link/?SJC/50/943/1.

[22] G. Hytönen, J. van Neerven, M. Veraar, and L. Weis, Analysis in Banach Spaces, Vol. 1: Martingales and Littlewood, Paley Theory, A Series of Modern Surveys in Mathematics, Ergebnisse der Mathematik und ihrer Grenzgebiete, 63 (2016), https://doi.org/10.1007/ 978-3-319-48520-1.

[23] K. Ito And K. Kunisch, Optimal control with $L^{p}(\mathcal{D}), p \in[0,1)$, control cost, SIAM Journal on Control and Optimization, 52 (2014), pp. 1251-1275, https://doi.org/10.1137/120896529.

[24] K. Ito And K. Kunisch, A variational approach to sparsity optimization based on Lagrange multiplier theory, Inverse Problems, 30 (2014), p. 015001, https://doi.org/10.1088/ 0266-5611/30/1/015001.

[25] D. Kouri, An Approach for the Adaptive Solution of Optimization Problems Governed by Partial Differential Equations with Uncertain Coefficients, PhD thesis, Rice University, 2012.

[26] D. P. Kouri, M. Heinkenschloss, D. Ridzal, and B. G. van Bloemen Waanders, A trust-region algorithm with adaptive stochastic collocation for PDE optimization under uncertainty, SIAM Journal on Scientific Computing, 35 (2013), pp. A1847-A1879.

[27] D. P. Kouri And T. M. Surowiec, Risk-averse PDE-constrained optimization using the conditional value-at-risk, SIAM Journal on Optimization, 26 (2016), pp. 365-396, https: //doi.org/10.1137/140954556.

[28] K. Kunisch, K. Pieper, And B. Vexler, Measure valued directional sparsity for parabolic optimal control problems, SIAM Journal on Control and Optimization, 52 (2014), pp. 30783108, https://doi.org/10.1137/140959055.

[29] J. Liu, S. Ji, AND J. YE, Multi-task feature learning via efficient $\ell_{2,1}$-norm minimization, CoRR, abs/1205.2631 (2012), https://arxiv.org/abs/1205.2631.

[30] K. Mohan And M. FAzel, Iterative reweighted algorithms for matrix rank minimization, The Journal of Machine Learning Research, 13 (2012), pp. 3441-3473, https://doi.org/10.1109/ ALLERTON.2010.5706969.

[31] F. Negri, G. Rozza, A. Manzoni, and A. Quarteroni, Reduced basis method for parametrized elliptic optimal control problems, SIAM Journal on Scientific Computing, 35 (2013), pp. A2316-A2340, https://doi.org/10.1137/120894737. 
[32] F. Nie, H. Huang, X. CAi, And C. H. Ding, Efficient and robust feature selection via joint $\ell_{2,1}$-norms minimization, in Advances in Neural Information Processing Systems 23, Curran Associates, Inc., 2010, pp. 1813-1821, http://papers.nips.cc/paper/ 3988-efficient-and-robust-feature-selection-via-joint-121-norms-minimization.pdf.

[33] K. Pieper, Finite element discretization and efficient numerical solution of elliptic and parabolic sparse control problems, Dissertation, Technische Universität München, 2015.

[34] Y. SAAD, Iterative methods for sparse linear systems, Society for Industrial and Applied Mathematics, Philadelphia, PA, second ed., 2003, https://doi.org/10.1137/1.9780898718003, https://doi.org/10.1137/1.9780898718003.

[35] G. STADLER, Elliptic optimal control problems with $L^{1}$-control cost and applications for the placement of control devices, Computational Optimization and Applications, 44(2) (2009), pp. 1-23, https://doi.org/10.1007/s10589-007-9150-9, http://www.springerlink. com/content/y6r1220572lk1530/.

[36] H. Tiesler, R. M. Kirby, D. Xiu, and T. Preusser, Stochastic collocation for optimal control problems with stochastic PDE constraints, SIAM Journal on Control and Optimization, 50 (2012), pp. 2659-2682. 